\title{
IDONEIDAD DISCIPLINAR DE LOS PROFESORES Y MERCADO DE HORAS DOCENTES EN CHILE ${ }^{1}$
}

\author{
María Paz Medeiros ${ }^{2}$ \\ Constanza Gómez ${ }^{3}$ \\ María Jesús Sánchez ${ }^{4}$ \\ Vanessa Orrego ${ }^{5}$
}

\section{RESUMEN}

El presente estudio describe la situación actual de los establecimientos educacionales chilenos en función de la idoneidad disciplinar de su planta docente y profundiza en sus mecanismos de selección y asignación de profesores. Además, estima la situación actual del mercado de horas aula y analiza el efecto que podría generar en la implementación del aumento de selectividad al ingreso de la carrera docente y el de horas no lectivas, definidos en la nueva Política Nacional Docente (PND). Las estimaciones indican que existe un número considerable de horas que no está siendo impartido por docentes con la especialidad correspondiente a la disciplina o nivel requerido y que, con el aumento de las horas no lectivas y la selectividad en las carreras de educación, se generaría un déficit crítico en el mercado de horas de clases de docentes si se mantienen las condiciones actuales, o bien menores déficits o superávit, en el caso de que aumente la atracción a la profesión debido a las mejores condiciones de la carrera docente. Finalmente, se generan recomendaciones que permitirían mejorar la implementación de estos importantes y necesarios cambios para la profesión docente.

Palabras clave: idoneidad disciplinar docente, mercado docente, oferta y demanda de horas.

\section{OUT-OF-FIELD TEACHING AND THE TEACHER LABOUR MARKET, IN CHILE}

\section{ABSTRACT}

The present study describes the out-of-field teaching situation, in Chile, and addresses in-depth the selection and assignment mechanisms of Chile's teachers. It estimates the current situation of the teacher's market and analyzes the effect that could result from the implementation of greater selectivity to enter the teaching profession and changes regarding non-teaching hours,

Proyecto apoyado financieramente por CNED/ Convocatoria 2016

Elige Educar, Santiago, Chile. Contacto: mmedeiros@eligeeducar.cl

Elige Educar, Santiago, Chile. Contacto: cgomez@eligeeducar.cl

Elige Educar, Santiago, Chile. Contacto: msanchez@eligeeducar.cl

Elige Educar, Santiago, Chile. Contacto: vorrego@eligeeducar.cl

Con especial agradecimiento, por su apoyo en distintas etapas del proyecto, a Juan Correa, Bernardita Crisóstomo, Hernán Hochschild, Juan Luis Cordero, David Inostroza y Pilar Riesco. 
as defined in the new education policy concerning teachers (Politica Nacional Docente, PND). The results indicate that there is still a considerable number of hours that are not being taught by teachers with the required specialty or level. The estimates further reveal that the proposed changes, for example, an increase in the proportion of non-teaching hours and greater selectivity in the initial teacher training programs could generate an impact on the supply and demand of teaching hours. If current conditions are maintained, the policy could produce a severe deficit scenario. If, however, the profession's attractiveness increases, due to improved conditions in the teaching profession, the scenario could be less dire and perhaps even accrue a surplus. Finally, we offer recommendations to improve the implementation of these important and necessary changes for the teaching profession.

Keywords: out-of-field teaching, supply and demand of teaching hours, teacher market.

\section{Introducción}

La educación es un desafío importante en Chile y las discusiones acerca del acceso, calidad y gratuidad han primado en la contingencia política y social. Gracias a la centralidad que han tomado los profesores en el debate, fue promulgada la Política Nacional Docente (PND, en adelante) en espera de mejorar su desarrollo profesional y condiciones laborales. Este hito abrió un nuevo escenario para la profesión docente que, entre otros, impone una mayor selectividad al ingreso a las carreras de educación y aumenta, para los docentes en aula, la proporción de horas no lectivas respecto de las lectivas.

En este contexto, resaltan dos temas a considerar dadas sus implicancias políticas y las reflexiones en torno al futuro de la profesión docente. El primero corresponde a la idoneidad disciplinar de los profesores y refleja la interrogante respecto del tipo de formación que poseen quienes están enseñando en aula. En este sentido, la literatura internacional ha profundizado en esta preocupación (Ee-gyeong, 2011; Galvis y Bonilla, 2011; Ingersoll, 1998; Jerald \& Ingersoll, 2002; Lu, Shen \& Poppink, 2007; Zhou, 2012), llegando incluso a consignar un impacto en el aprendizaje de los estudiantes y en la rotación y deserción de los profesores (Ingersoll, 1998), o que se trata, al menos, de uno de los factores involucrados (Clotfelter, Ladd, Vigdor \& Wheeler, 2007; De Angelis, Presley \& White, 2005; Kane, Rockoff \& Staiger, 2006). En Chile, por ejemplo, la relevancia del dominio disciplinar de la asignatura 
que enseña el docente ha sido explicitada en el Marco para la Buena Enseñanza e incentivada por la Bonificación de Reconocimiento Profesional de Mención (BRP), desde donde ha sido vinculada con la calidad de la docencia. La promulgación del Decreto 325 de la Ley $\mathrm{N}^{\circ} 18.956$ complementa estas iniciativas, fijando la formación mínima para todos los docentes de aula y, además, estableciendo las especializaciones requeridas en educación media.

Un elemento crítico de la idoneidad disciplinar es la distribución de los docentes en el sistema educativo y, por tanto, se encuentra estrechamente relacionada con el mercado docente y las lógicas que operan tras la oferta y demanda de profesores, que es un segundo elemento relevante de considerar al analizar la implementación de la PND. En concreto, se estima el número de horas que los docentes ofrecen actualmente en el sistema, y cuántas de ellas y dónde se necesitarán a partir de la PND.

A diferencia de la idoneidad disciplinar, el estudio de la oferta y demanda de docentes no es algo nuevo en Chile. Las investigaciones han incorporado, por un lado, las trayectorias, movilidad y abandono de los profesores (Cabezas, Paredes, Bogolasky, Rivero \& Zarhi, 2017; Fuenzalida, Ávalos, Valenzuela y Acuña, 2014; Valenzuela y Sevilla, 2013) y, por otro, su dotación y los escenarios que podrían afectarla (De Hek, De Jong y De Koning, 2017; Montoya, 2005; Montoya y Blackburn, 2010; Sánchez et al., 2013; Sepúlveda, 2015). El análisis de Sánchez et al. (2013) reportó un superávit de docentes en Inglés, Educación Física e Historia, mientras en Física y Educación Básica proyectaron escasez. Este escenario se ve necesariamente modificado por las políticas educativas en curso. Así, un análisis posterior de Gutiérrez, Medeiros y Sánchez (2013) acerca del efecto de la Beca Vocación de Profesor (BVP) en las proyecciones estimadas en Sánchez et al. (2013), determinó que esta representó un impacto marginal respecto del superávit de docentes, excepto en las especialidades con déficit. A propósito del aumento en los requisitos de selección a la carrera de Pedagogía, se ha señalado una reducción en el ingreso de los estudiantes (Centro de Investigación Avanzada en Educación, CIAE, 2017), principalmente en las universidades privadas no adscritas al Sistema Único de Admisión (SUA) (Elige Educar, 2017), 
lo que sin duda tendrá un efecto en el mercado docente. Sin embargo, aún no se han revisado en profundidad sus implicancias, ni tampoco el aumento de las horas no lectivas y sus proyecciones en el mercado docente, especialmente si se considera la idoneidad disciplinar.

Tomando en consideración lo anterior, el presente estudio avanzará en responder la siguiente pregunta: ¿Cómo se distribuye la idoneidad disciplinar y el mercado de horas docentes presente y futuro en Chile? En lo concreto, el objetivo general de la investigación es describir la idoneidad disciplinar de los docentes en los establecimientos educacionales del país y estimar la oferta y demanda de horas docentes actual y futura a partir de la implementación de la PND. En tanto que sus objetivos específicos son los siguientes:

- Describir la idoneidad disciplinar de los establecimientos educacionales según sus características administrativas, pedagógicas, docentes y de nivel o grupo socioeconómico (GSE).

- Describir el comportamiento del mercado de horas docentes actual y futuro por región, nivel de enseñanza y grado, considerando los posibles efectos del aumento de las horas no lectivas y de los requisitos de ingreso a la carrera de educación.

- Comprender los factores intra y extraescuela que podrían facilitar u obstaculizar los cambios implementados por la PND en idoneidad disciplinar y mercado de horas docentes.

- Proveer recomendaciones asociadas a la atracción y movilidad de docentes para orientar la implementación de la PND.

\section{Revisión de literatura}

\section{1. Idoneidad disciplinar: out-of-field teaching}

Numerosos estudios concluyen que los profesores son el factor intraescuela más importante en el logro de aprendizaje de sus alumnos (Barber y Mourshed, 2008; Sanders \& Rivers, 1996). Un buen profesor puede generar que sus estudiantes aprendan un 50\% o 100\% más que sus pares, medido a través de pruebas estandarizadas (Bloom, Hill, Black \& Lipsey, 2008; Hanushek \& Rivkin, 2010). Este impacto depende de diversos conocimientos, habilidades y 
destrezas del profesor, los cuales han sido medidos indirectamente a través de las certificaciones de pregrado, calidad de las instituciones formadoras, resultado de pruebas de evaluación docente y años de experiencia profesional, entre otros (Clotfelter et al., 2007).

En Chile, el Marco para la Buena Enseñanza (Ministerio de Educación de Chile, Mineduc, 2008) sirve como guía para ayudar a determinar la calidad de la docencia, ya que especifica tres competencias esenciales que requiere todo profesor:

- dominio del contenido disciplinar,

- dominio de la didáctica del área que enseñan y

- habilidades socioemocionales que le permitan construir un clima de aula que promueva el aprendizaje.

La falta de idoneidad disciplinar (out-of-field-teaching, en inglés), se define como el fenómeno por el cual un profesor enseña una determinada asignatura sin tener la especialidad y/o certificado afín (Ingersoll \& Gruber, 1996). Se refiere, por ejemplo, al docente que posee mención en Historia y realiza clases de Ciencias o Matemática; o a aquel profesional que está titulado para ejercer en enseñanza media y que realiza clases en el primer ciclo de educación básica. La definición exacta dependerá de los requisitos impuestos por cada país (Zhou, 2012), pudiendo ir desde contar con el título de profesor hasta la exigencia de un postítulo o tener una certificación especial (Ingersoll, 2001).

En la década de los noventa, Estados Unidos ya señalaba su preocupación: 35\% de las clases de Matemática eran enseñadas por profesores sin minor en la temática o en un área relacionada y cerca de la mitad de las clases de Ciencias Naturales y Ciencias Sociales eran impartidas por docentes sin certificado para enseñar dichos contenidos (Ingersoll, 1998). En Corea del Sur, por su parte, los problemas de idoneidad en Ciencias y Matemática afectaban al $23 \%$ y $10 \%$ de los docentes, respectivamente (Ee-gyeong, 2011), mientras que en Colombia, concluyen Galvis y Bonilla (2011), solo el $77 \%$ de los profesores ha cursado carreras relacionadas con la pedagogía. 
La comparación internacional, a través del análisis de la encuesta TALIS, de las asignaturas de Matemática y Ciencias de escuelas públicas de 21 países confirma lo común que es la falta de idoneidad disciplinar - $10 \%$ en promedio- Se destacan problemas principalmente en escuelas rurales, con menor número de alumnos y de menor nivel socioeconómico (Zhou, 2012), considerándose en este último grupo a las comunidades minoritarias (Castro \& Esposito, 2017; Jerald \& Ingersoll, 2002; Lu et al., 2007; Schultz, 2014).

Los establecimientos educacionales de menor grupo socioeconómico (GSE) y menor idoneidad también tienden a concentrar a los profesores que provienen de instituciones menos competitivas, sin licencias regulares, con menor puntaje en las pruebas de desempeño docente (Clotfelter et al., 2007), menor experiencia profesional (Ingersoll, 1998; Nixon, Luft \& Ross, 2017; Zhou, 2012) y sin cursos de posgrado (Galvis y Bonilla, 2011). En estas escuelas, los problemas se concentran, además, en las disciplinas con mayor carga horaria, tales como Matemática, Lenguaje y Ciencias (Ingersoll, 1998; Jerald \& Ingersoll, 2002). De esa manera, las escuelas que más necesitan profesores altamente calificados son las que tienden a presentar menor número de los mismos (Rivero, 2015). Se ha evidenciado una relación con el nivel de autonomía del sistema educativo, específicamente con su capacidad para tomar decisiones de contrataciones y manejo del presupuesto para la definición de salarios y desarrollo profesional de sus docentes (Zhou, 2012).

Otros patrones dicen relación con el tamaño del establecimiento, donde existe una mayor tendencia a tener profesores no especializados en escuelas pequeñas (Ee-gyeong, 2011; Jerald $\&$ Ingersoll, 2002; Zhou, 2012), debido probablemente a que no existe una cantidad suficiente de profesores para cubrir todas las asignaturas. Por último, respecto de la dependencia administrativa, si bien no se observa una tendencia clara, distintos estudios reiteran diferencias entre establecimientos en su capacidad de atraer a docentes (Eegyeong, 2011; McConney \& Price, 2009; Rivero, 2015).

Si bien no existe suficiente evidencia para indicar causalidad, sí se ha descrito una relación entre la falta de idoneidad disciplinar 
docente y los aprendizajes de los estudiantes: por ejemplo, los cursos que suelen estar bajo el alero de un docente no certificado en su área de especialización obtienen resultados académicos más bajos que las otras asignaturas (Ingersoll,1998). La gradualidad de este impacto, sin embargo, dependerá también de otros factores. De Angelis et al. (2005) recalcan que los recursos monetarios y la calidad del liderazgo pedagógico pueden intensificar o aminorar la calidad de la enseñanza del profesor sobre los estudiantes. La experiencia de un docente juega también un rol central, señala Knoeppel (2007). En tanto que Kane et al. (2006) concluyen que no existen diferencias estadísticas entre un docente novato con certificación y un profesor sin ella, pero con múltiples años de experiencia. Sin embargo, esto no implica que la selección —al momento de la contratación — sea irrelevante, pues deben medirse también las habilidades del docente para la enseñanza.

\subsection{Mercado de horas aula (MHD)}

El MHD se ha convertido en un foco de atención para diversos sistemas educativos, debido a los altos índices de deserción del sistema (Organisation for Economic Co-operation and Development, OECD, 2005). Al estudiar el mercado de horas aula se debe tener en cuenta que el equilibrio se genera cuando la cantidad de horas ofrecidas es igual a la cantidad de horas necesarias para cubrir las salas de clases; mientras que el déficit ocurriría cuando existe mayor demanda que oferta. Sin embargo, en términos estrictos, no existen clases que se realicen sin profesores. Dos escenarios permiten cuantificar el desequilibrio: vacantes que no se pueden llenar (vacancy rates) y la escasez escondida (hidden shortages), lo cual refiere a profesores dictando asignaturas sin tener la preparación suficiente (Jackson, 2009; Santiago, 2002).

Durante los últimos años, se han realizado una serie de estudios que abordan el tema. Por un lado, Santiago (2002) propone un marco de análisis que relaciona la estructura del mercado (gobernanza del sistema escolar, regulación, entre otros), oferta potencial (qué tan atractiva es percibida la profesión mediante salarios, condiciones laborales, estructura de la carrera, etc.) y calidad de la docencia (certificación, evaluación, experiencia, entre otros). Por otra parte, se 
ha estudiado en términos de horas de docencia requeridas (Montoya y Blackburn, 2010) y según el efecto de diferentes escenarios de horas no lectivas (Sepúlveda, 2015). A lo largo de estas investigaciones, se visualiza un panorama de superávit general de profesores, el cual varía por especialidad, región y nivel educativo. En particular, bajo un escenario de aumento de horas no lectivas, Sepúlveda (2015) proyecta un posible déficit de docentes en las regiones chilenas de Antofagasta, O’Higgins y el Maule y en especialidades como Ciencias.

Este fenómeno obliga a comprender complejamente el MHD, para dar cuenta del número de profesores existentes y necesarios en el marco de sus especializaciones y distribución de la jornada laboral, procesos en transformación gracias a la PND.

\section{Enfoque metodológico}

La investigación corresponde a un estudio mixto donde se aplicó tanto análisis cuantitativo como cuantitativo de los datos.

\subsection{Análisis cuantitativo}

Comprendió la construcción de un indicador de idoneidad disciplinar docente por establecimiento para el año 2016 y la proyección del mercado docente, en términos de horas docentes, para el período 2017-2031, a partir de la situación de 2016. Se utilizaron bases de datos públicas disponibles en el sitio web del Ministerio de Educación:

- Idoneidad Docente (2003-2016),

- Matrícula Única Oficial (2004-2016),

- Rendimiento por Alumno (2002-2015),

- Directorio de Establecimientos (2016) y

- Seleccionados por Asignación por Desempeño Difícil (2016).

Además, se recurrió a información publicada por el Servicio de Información de Educación Superior (SIES): Matrícula 2007-2016 y Titulados 2007-2016; por Mi Futuro: Estadísticas por Carrera (2017); y por el Consejo Nacional de Educación (CNED): Índices BD Matrícula 2005-2017. 


\subsection{Indicador de idoneidad disciplinar docente}

Se construyó un indicador que reflejara la proporción de horas de clases por establecimiento -impartidas por docentes cuya especialidad era idónea para la asignatura que realizaban-, respecto del total de horas de clase impartidas en el establecimiento en cierta asignatura y/o nivel de enseñanza. Los criterios utilizados siguieron lo señalado en el Decreto 352 de la Ley 18.956, elevando las restricciones en algunos casos. Se consideró idóneo al docente con título de Educación de Párvulos y Pedagogía Básica, que realizaba clases en el nivel respectivo, independientemente de la asignatura que impartiera. Mientras en enseñanza media, además del título general de Pedagogía Media, se solicitó contar con la especialidad de la asignatura que impartía. Por lo tanto, el indicador contemplado fue de $0 \%$ a $100 \%$, donde $0 \%$ reflejaba que ninguna de las horas aula impartidas en el establecimiento fue realizada por docentes idóneos y 100\%, que implicó que todas las horas aula fueron realizadas por estos docentes.

\subsection{Mercado de horas docentes 2016 y proyección para 2017-2031}

Las estimaciones se realizaron tomando en cuenta cargos docentes ${ }^{7}$, cuya función principal o secundaria era la docencia en aula en educación de párvulos (NT1 y NT2), básica, media científicohumanista (C-H) y media técnico-profesional (T-P), excluyendo la enseñanza de adultos y especial.

La caracterización del mercado docente para el año 2016 se basó en los estudios desarrollados por Montoya (2005), Montoya y Blackburn (2010) y Sánchez et al. (2013). Se estimó la oferta de horas aula por asignatura, nivel de enseñanza y región, considerando las horas de clase reportadas por cada docente. Se asumió que el 25\% de esas horas eran no lectivas. La demanda se estimó a partir del plan de estudios con el que debe cumplir cada establecimiento según lo exigido por Mineduc y el número de cursos existentes al año 2016

El cargo docente se refiere al contrato entre docente y establecimiento. Es decir, un docente puede tener más de un cargo docente si trabaja en más de un establecimiento. 
(para mayor detalle ver Medeiros, Gómez, Orrego y Sánchez, 2017). Una vez obtenida la oferta y demanda vigentes para el año 2016, se calculó el superávit (o déficit) de horas aula ${ }^{8}$, por asignatura, nivel de enseñanza y región de Chile.

Para la proyección de Mercado Docente 2017-20319, se estimó la oferta considerando las horas ofrecidas el año anterior, descontando las horas perdidas por docentes que desertaron del sistema o se jubilaron, y agregando las horas impartidas por aquellos que ingresaron por primera vez ${ }^{10}$.

Para proyectar la tasa de deserción, según Valenzuela y Sevilla (2013), se considera que un docente se retira del sistema educacional cuando figura en alguna Base de Idoneidad Docente previa a 2012 y luego no vuelve a aparecer hasta el año 2016. A diferencia de estos autores, para catalogar su situación como "abandono de la profesión" se contempló como mínimo cinco años de desaparición de las bases de datos, obteniendo el número de docentes que desertaron para el período 2004-2012 11 .

Para proyectar la tasa de jubilación se calcularon las horas de docencia de los profesores hombres mayores de 65 años y de las mujeres mayores de 60 que aparecían en las Bases de Idoneidad Docente previas a 2016, pero que no lo hacían en los siguientes dos

3. Como se señaló en el marco teórico, no debiesen existir alumnos sin profesor, es decir, el mercado debiera estar en equilibrio (oferta igual a demanda docente). Sin embargo, esta situación de equilibrio se puede producir en un escenario donde alguno de los supuestos planteados por el presente estudio no se estén cumpliendo. Por ejemplo, la estimación de esta investigación considera que todos los establecimientos cumplen con los planes de estudio y bases curriculares del Mineduc. Sin embargo, cuando esto no es así en la realidad, la estimación arrojará un desequilibrio que puede ser un déficit de horas docentes (si el establecimiento hace menos horas de clases de lo que indican las bases curriculares) o superávit (si el establecimiento hace más horas).

$9 \quad$ La Base de Idoneidad Docente permite a los directivos reportar horas docentes solamente en dos actividades/asignatura. Así, los valores pueden estar levemente subestimados al no considerar a los docentes que realizan más de dos asignaturas.

10 Por limitación de los datos no se consideró la oferta de horas aula potencial proveniente de los docentes que se encuentran fuera del sistema y que estarían dispuestos a regresar.

11 El último año para el que se puede estimar la tasa de deserción es 2012. Los docentes que aparecieron en la base de datos del año 2011 y que luego no figuraron en las bases de datos de los próximos cinco años, es decir, de 2012 a 2016, se asumió que desertaron, es decir, que terminaron el año escolar el 2011 y desertaron en 2012. 
años. Así mismo, para proyectar las horas de docentes que entraron en el sistema, se estimó la tasa de matrícula de la carrera de Pedagogía, así como la tasa de titulación y la de empleabilidad. Para proyectar la tasa de crecimiento de la matrícula en las carreras de educación se tuvieron en cuenta dos aspectos de la Política Nacional Docente detallados en las Tablas 1 y 2 .

Tabla 1

Proporción horas lectivas y no lectivas según PND

\begin{tabular}{lcccc}
\hline Año de cumplimiento & Situación inicial [1] & 2017 [2] & 2019 & 2027 \\
\hline $\begin{array}{l}\text { Horas lectivas/ } \\
\text { Horas no lectivas }\end{array}$ & $75 / 25$ & $70 / 30$ & $65 / 35[3]$ & $60 / 40[4]$ \\
\hline
\end{tabular}

[1] A partir del año 2016 la Base de Idoneidad Docente del Ministerio de Educación comenzó a reportar horas de clases cronológicas, en vez de pedagógicas, sin especificar la inclusión de las horas no lectivas. Luego de analizar varios escenarios, se optó por considerar estas horas reportadas como horas aula totales, asumiendo que incluían tanto al $75 \%$ de las horas lectivas como al $25 \%$ horas no lectivas.

[2] De acuerdo con la Ley 20.903, para el año 2019 se debe cumplir la proporción respectiva. Sin embargo, para el resto de los años, las proporciones supuestas, son propias. [3] Para los profesores de $1^{\circ}$ a $4^{\circ}$ año básico en colegios que tienen más del $80 \%$ de alumnos prioritarios, la proporción para el año 2019 será de 60/40.

[4] El 60/40 para los docentes (excepto los de $1^{\circ}$ a $4^{\circ}$ año básico en colegios que tienen más del $80 \%$ de alumnos prioritarios) está sujeto a elevados crecimientos de la economía del país. Por lo que se debe tener en cuenta que no necesariamente es una exigencia para los establecimientos por parte del Mineduc.

Fuente: Elaboración propia.

Tabla 2

Criterios de selección a las carreras de Pedagogía

\begin{tabular}{|c|c|c|c|}
\hline $\begin{array}{c}\text { Año de } \\
\text { cumplimiento }\end{array}$ & 2017 & 2020 & 2023 \\
\hline \multirow{9}{*}{ Requisitos } & 500 PSU & 525 PSU & $550 \mathrm{PSU}$ \\
\hline & $\mathrm{O}$ & $\mathrm{O}$ & $\mathrm{O}$ \\
\hline & 30\% Ranking & 20\% Ranking & 10\% Ranking \\
\hline & $\mathrm{O}$ & & $\mathrm{O}$ \\
\hline & PSU rendida + & $40 \%$ Ranking +500 & 30\% Ranking + 500 \\
\hline & PSU rendida + & PSU & PSU \\
\hline & Programa de acceso & $\mathrm{O}$ & $\mathrm{O}$ \\
\hline & & PSU rendida + & $\begin{array}{l}\text { Programa de acceso + } \\
15 \% \text { Ranking }\end{array}$ \\
\hline & & Programa de acceso & \\
\hline
\end{tabular}

Fuente: Elaboración propia basada en la Ley No 20.903. 
Para proyectar el cambio en la matrícula, se consideró el crecimiento anual promedio del período 2011 y $2016^{12}$, excluyendo de este cálculo a los matriculados en instituciones de educación superior que no estaban vigentes al 2017. La tasa fue de 1,65\%. A este crecimiento a tasa constante se le aplicaron modificaciones en los años 2020 y 2023 , debido al aumento del puntaje de corte PSU a 525 y 550 , correspondientemente ${ }^{13}$. Las modificaciones ocasionadas por el aumento del puntaje de corte PSU se basaron en la distribución de puntajes PSU de matriculados en carreras de Pedagogía el $2015^{14}$ según especialidad e institución. Una vez asumida la distribución, se aplicaron los parámetros que existían para el 2017 (mínimo, máximo, $\mathrm{N}$ total y promedio del puntaje PSU de los matriculados a carreras de Pedagogía por institución) y se dividió en ocho partes, donde cada una obtuvo el 10\% de las observaciones en sus colas y $80 \%$ cerca de las medias.

Para la proyección, en los escenarios más negativos (escenarios 1 y 2 de la Tabla 4), se asumió que los parámetros de 2017 quedaban constantes al cambio en los puntajes de corte, es decir, que no existía un aumento de matriculados en carreras de Pedagogía generado por un mayor interés en el área, debido a una mayor selectividad. Por lo tanto, para el año 2020 se tomó el puntaje mínimo de los matriculados para cada Pedagogía e institución. Si este era mayor a 525 puntos, entonces se asumió que la matrícula en ese lugar crecería a la tasa promedio que venía haciéndolo durante el periodo 2015 y 2016. Por otra parte, si el puntaje mínimo era menor a 525, se evaluó el siguiente máximo de la siguiente parte de la distribución: si este no superaba los 525, se asumió que el 10\% más bajo de la distribución quedaba fuera, y así sucesivamente.

12 Se excluyó la tasa de crecimiento del 2017, debido a que ese año comenzó a regir la PND y se produjeron crecimientos irregulares de la matrícula según institución y Pedagogía. Este cambio se consideró luego para los años en donde se implementaron las medidas de PND relacionadas con modificaciones en el puntaje mínimo PSU necesario.

13 No se tomaron en cuenta los cambios en requisitos de ingreso a Pedagogía relacionados con ranking de notas y programa de acceso a la educación superior reconocido por el Mineduc, porque no se contaba con la información del número de alumnos correspondientes. Eso podría implicar un aumento en la matrícula de cada año.

14 No se tienen los datos individuales de puntaje PSU de los matriculados en Pedagogía. 
En los escenarios más positivos (Tabla 4 , escenarios 3 y 4) se consideró que la matrícula crecería en función del aumento del interés por estudiar Pedagogía, dadas las mejores condiciones en remuneraciones y mayor selectividad de la carrera. Un estudio realizado por Elige Educar (2014) utilizando la metodología "conjoint" determinó que en una muestra de 183 egresados de cuarto medio, las preferencias por estudiar Pedagogía aumentaban hasta en un $37 \%$ si cambiaban algunas condiciones como la selectividad de la carrera y el salario. La Tabla 3 reporta los resultados obtenidos por ese estudio.

Tabla 3

Resultados obtenidos por Elige Educar (2014)

\begin{tabular}{lccc}
\hline $\begin{array}{c}\text { Opciones de política } \\
\text { acerca de las carreras } \\
\text { de educación }\end{array}$ & $\begin{array}{c}\text { Filtro de } \\
\text { selección }\end{array}$ & $\begin{array}{c}\text { Remuneración } \\
\text { promedio }\end{array}$ & $\begin{array}{c}\text { Preferencia } \\
\text { por carreras de } \\
\text { educación }\end{array}$ \\
\hline Opción 1 & $\begin{array}{c}\text { Sin puntaje de corte } \\
\text { Opción 2 }\end{array}$ & $\$ 500.000$ & $12,4 \%$ \\
Opción 3 & 500 puntos PSU & $\$ 500.000$ & $13,9 \%$ \\
Opción 4 & 500 puntos PSU & $\$ 750.000$ & $21,7 \%$ \\
Opción 5 & 500 puntos PSU & $\$ 1.000 .000$ & $29,4 \%$ \\
Opción 6 & 550 puntos PSU & $\$ 1.000 .000$ & $34,7 \%$ \\
\hline
\end{tabular}

Fuente: Elige Educar, 2014.

Puesto que las condiciones de la PND se aproximan a las opciones 3 y 5 del estudio Elige Educar (2014), se asumió que el año 2018 la matrícula aumentará en 21,7\%; y que al llegar a 2023, esta se habrá incrementado en un 34,7\% (asumiendo una tasa de crecimiento anual constante de 2,6\%).

La información de matrícula se consideró con un rezago de cinco períodos dado que en promedio la carrera tiene una duración de cinco años. Este cálculo se realizó por subgrupos, tomando en cuenta la región, nivel de enseñanza y especialidad de la carrera de Pedagogía. Se hizo el mismo ejercicio para la tasa de titulación, a partir de la Base de Datos de Titulados del SIES, obteniendo una tasa de 0,7296645 que se aplicó para todas las carreras y, finalmente, a partir de la Base de Datos Estadísticas por Carrera del SIES se logró 
la empleabilidad, esto es, la probabilidad de que el recién titulado encuentre un empleo el primer año o el segundo luego de concluida la carrera. Los docentes que entran al sistema educacional por primera vez, lo hacen teniendo un número de horas aula que, en promedio, han tenido los que han ingresado al sistema en la misma región y habiéndose titulado de la misma Pedagogía.

Para el estudio, las horas aula demandadas futuras están dadas por la matrícula estimada futura, el tamaño de los cursos y el Plan de Estudios del Mineduc que se asume constante para el período de proyección. Ambas medidas se estimaron a nivel de región, asignatura y nivel de enseñanza. La matrícula futura de los colegios estuvo dada por la matrícula del año anterior, ajustándose por la tasa de reprobación y la tasa de deserción. La tasa de reprobación, en tanto, se obtuvo de la base de datos Rendimiento por Alumno, calculando el promedio de reprobación para los años disponibles, y se supuso fija para los años de proyección ${ }^{15}$. Respecto de la tasa de deserción escolar, se asumió que un alumno deserta del sistema escolar si es que aparece en las bases de datos de Matrícula y luego desaparece sistemáticamente para los años posteriores, excluyendo a los alumnos que cursan cuarto medio. Luego, para estimar el tamaño de los cursos, la investigación se basó en Montoya y Blackburn (2010), esto es, se proyectó la razón de alumnos por curso, según el promedio del tamaño de cursos obtenido para período 2003-2016, por grado, asumiendo que esta se mantendría constante para el período 20172031. Finalmente, se supuso que el plan de estudios vigente para el año 2016, también se mantendría constante para el año 2031.

La segunda dimensión de la PND que se consideró en esta proyección fue el cambio en la proporción de horas lectivas y no lectivas. Para incluirla en el análisis se partió del supuesto que todos los docentes tenían una proporción de 0,75 de horas lectivas y 0,25 de horas no lectivas en 2016, según lo reportado en las Bases de Idoneidad Docente (Mineduc). Teniendo esto en cuenta, la oferta total de horas mensuales para dicho año fue de 15.865.207.

15 La cantidad de años para la cual se tome el promedio dependerá de la varianza de la tasa de reprobación a través de los años. 
A partir de esto, al número de horas de clases se le descontó la proporción respectiva cada año para llegar a la proporción exigida por la PND. En un escenario negativo (escenarios 1 y 3 de la Tabla 4) se supuso que esta disminución no será cubierta por nuevas horas de docentes. En el escenario positivo (escenarios 2 y 4 de Tabla 4) se asumió que la disminución podría ser cubierta por los docentes dentro del sistema escolar que aumentaran sus horas de contrato (si ello es posible debido a que tienen menos de 44 horas semanales de contrato) y por aquellos nuevos que entraran al sistema escolar y que podrían aumentar sus horas de contrato alcanzando las 44 horas semanales.

Tabla 4

Escenarios a considerar en el estudio según la reacción del mercado ante la PND

\begin{tabular}{lcc}
\hline Matrícula & $\begin{array}{c}\text { No se producen cambios } \\
\text { en las horas aula de } \\
\text { los docentes nuevos y } \\
\text { existentes en el sistema }\end{array}$ & $\begin{array}{c}\text { Aumento de horas aula } \\
\text { de docentes nuevos y } \\
\text { existentes en el sistema }\end{array}$ \\
\hline $\begin{array}{l}\text { Disminución de matrícula } \\
\text { no contrarrestada }\end{array}$ & Escenario 1 & Escenario 2 \\
\hline $\begin{array}{l}\text { Disminución de matrícula } \\
\text { contrarrestada por aumento } \\
\text { del interés }\end{array}$ & Escenario 3 & Escenario 4 \\
\hline
\end{tabular}

Fuente: Elaboración propia.

Finalmente, el equilibrio se obtiene de restar la oferta y la demanda por año, por región, asignatura y nivel de enseñanza.

\subsection{Análisis cualitativo}

Se realizaron siete entrevistas semiestructuradas a directores de establecimientos educacionales, las cuales permitieron introducir temas adicionales no considerados previamente (Hernández, Fernández y Baptista, 2010). El muestreo fue intencionado por dependencia administrativa, región y nivel de idoneidad disciplinar de los docentes ${ }^{16}$.

16 Para el aspecto referido a la región se consideraron solo las de Atacama, Valparaíso y Metropolitana, ya que reflejan las mayores variaciones de idoneidad a nivel nacional; mientras que respecto de idoneidad se consideró el indicador construido en el presente trabajo y la comparabilidad según dependencia y región. 
Tras la selección, se organizó una entrevista con aquellos directores que estaban interesados en participar y podían hacerlo entre agosto y la primera quincena de septiembre de $2017^{17}$ y se procedió a su transcripción y codificación. Se utilizó la codificación axial a través de rejillas, empleando el software Excel.

\section{Resultados}

\subsection{Análisis cuantitativo}

3.1.1. Idoneidad disciplinar docente según nivel de enseñanza, dependencia administrativa y asignatura

El análisis de los docentes que hoy ejercen en el sistema educativo chileno revela que, en promedio, el 92\% de las horas impartidas en cada establecimiento escolar en el nivel de educación de párvulos y el $85 \%$ de las horas de enseñanza básica es realizada por docentes que cumplen con los criterios aplicados. La situación es compleja en enseñanza media, nivel donde se requiere contar con especialización: solo el 78\% de las horas es realizado por docentes con idoneidad disciplinar (Gráfico 1). La diferencia entre los niveles es significativa con un $99 \%$ de confianza.

17 Las invitaciones, realizadas por teléfono o correo electrónico y en contacto directo con los directores, comprendieron la indicación acerca de los objetivos y relevancia de la investigación y el procedimiento de recolección de datos, remarcando su confidencialidad. Las entrevistas fueron realizadas por el equipo investigador de Elige Educar, todos profesionales de las ciencias sociales, y en solo una visita a los establecimientos educacionales. 


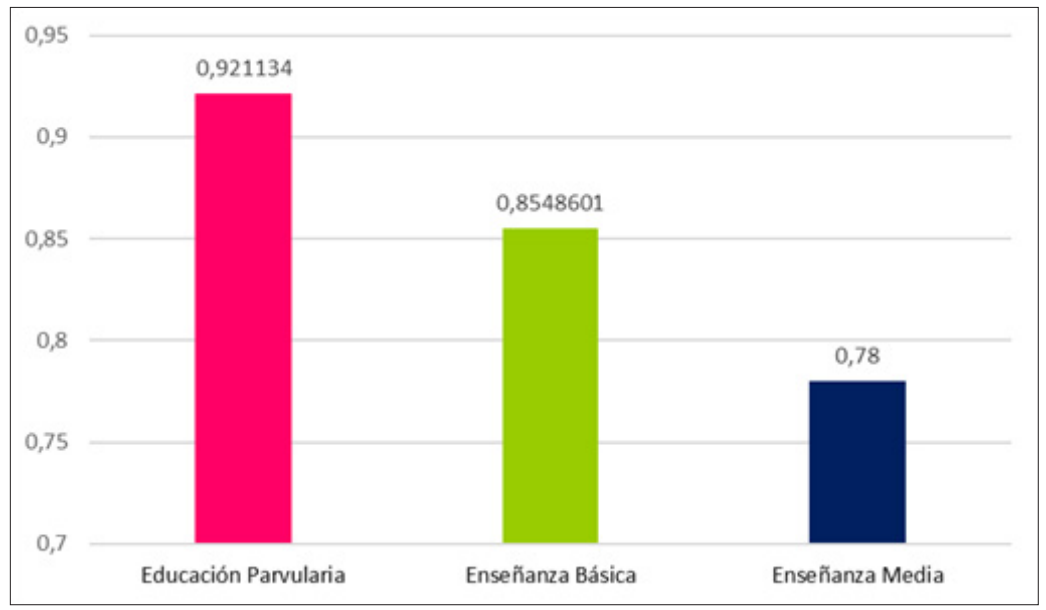

Gráfico 1. Idoneidad disciplinar por nivel de enseñanza.

Fuente: Elaboración propia a partir de la Base de Idoneidad Docente 2016, Mineduc.

El análisis por dependencia administrativa reportó mayor idoneidad disciplinar en los establecimientos particulares subvencionados en relación con los municipales y particulares pagados, siendo la diferencia significativa en un 90\% de confianza en ambos casos (ver Gráfico 2). Al considerar solo enseñanza media, esta distribución cambió y los particulares pagados aparecen con mayores tasas de idoneidad que los particulares subvencionados, seguidos por los municipales. Según Rivero (2015), a mayor especialización del docente es más probable que este haga clases en establecimientos de mayor ingreso y nivel académico. 


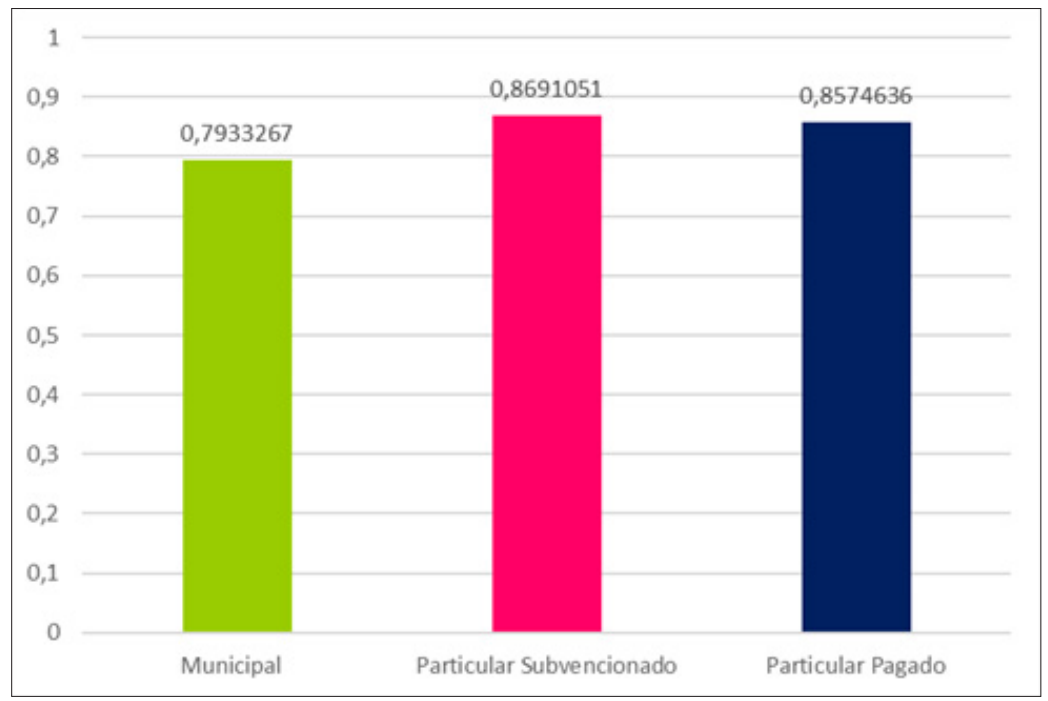

Gráfico 2. Idoneidad disciplinar total según dependencia.

Fuente: Elaboración propia a partir de la Base de Idoneidad Docente 2016, Mineduc.

Tal como muestra la literatura internacional (Nixon et al., 2017; Polizzi, Jaggernauth, Ray, Callahan \& Rushton, 2015), existen diferencias en el nivel de idoneidad disciplinar entre enseñanza básica y media. En enseñanza básica, las asignaturas con menor nivel de idoneidad son Tecnología (60\%), Lenguaje y comunicación (80\%) y Religión (89\%) $)^{18}$; en las dos primeras, los establecimientos municipales presentan una menor tasa. Inversamente, Ciencias es la asignatura con mayor nivel de idoneidad $(96 \%)^{19}$, siendo la dependencia municipal la administración con la tasa más alta.

En enseñanza media, Física (52\%), Ciencias Naturales (60\%), Química (61\%) y Biología (66\%) presentaron el menor nivel de idoneidad; mientras que Historia y Ciencias Sociales (95\%) e Inglés (95\%) obtuvieron las más altas tasas (ver Gráfico 3). Cuando se compara entre dependencias, se encuentran diferencias significativas

18 Lengua indígena es una de las asignaturas con menor nivel de idoneidad; sin embargo, la construcción de este aspecto subestima la realidad, debido a que existen profesores que, sin tener el título de Pedagogía, están habilitados por el Mineduc para impartir clases en la asignatura.

19 Esta asignatura incluye Comprensión del medio social y cultural, Estudio y comprensión de la naturaleza, Estudio y comprensión de la sociedad y otras asignaturas similares. 
solo entre municipales (73\%) y particulares pagados (78\%). Las mayores diferencias entre establecimientos municipales y particulares subvencionados se produjeron en Física, Química y Ciencias Naturales.

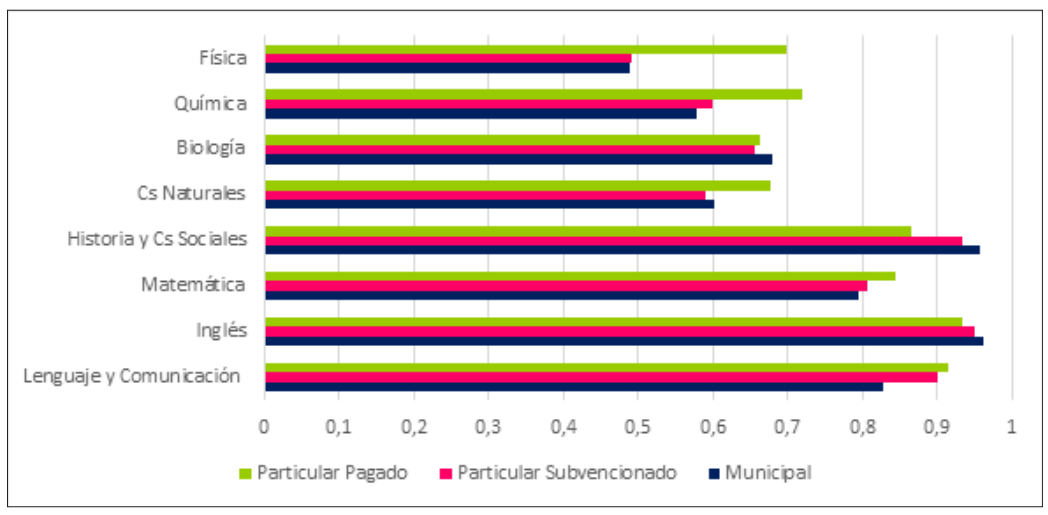

Gráfico 3. Tasas de idoneidad disciplinar enseñanza media, según dependencia.

Fuente: Elaboración propia a partir de la Base de Idoneidad Docente 2016, Mineduc.

\subsubsection{Idoneidad disciplinar docente según región y ruralidad}

El análisis regional evidenció diferencias interesantes por nivel. En educación inicial, las regiones de Aysén (88\%) y Biobío (90,43\%) reportaron menor idoneidad disciplinar; mientras que Arica y Parinacota presentó la tasa más alta $(97,71 \%)$. En enseñanza básica, las tasas más bajas las obtuvieron Los Ríos (81,93\%), Arica y Parinacota (90,05\%), Maule (83,77\%) y Biobío (79,78\%), mientras que Arica y Parinacota $(90,05 \%)$ presentó el índice más alto del país. Finalmente, en enseñanza media la región de Atacama presentó la idoneidad disciplinar más baja (66,6\%) junto con Tarapacá $(67,06 \%)$, mientras que Arica y Parinacota (86,08\%) tuvo la más alta (Gráfico 4). De estos resultados se desprende que la problemática de idoneidad disciplinar se concentra, principal y uniformemente en educación media en el área de Ciencias Naturales. 


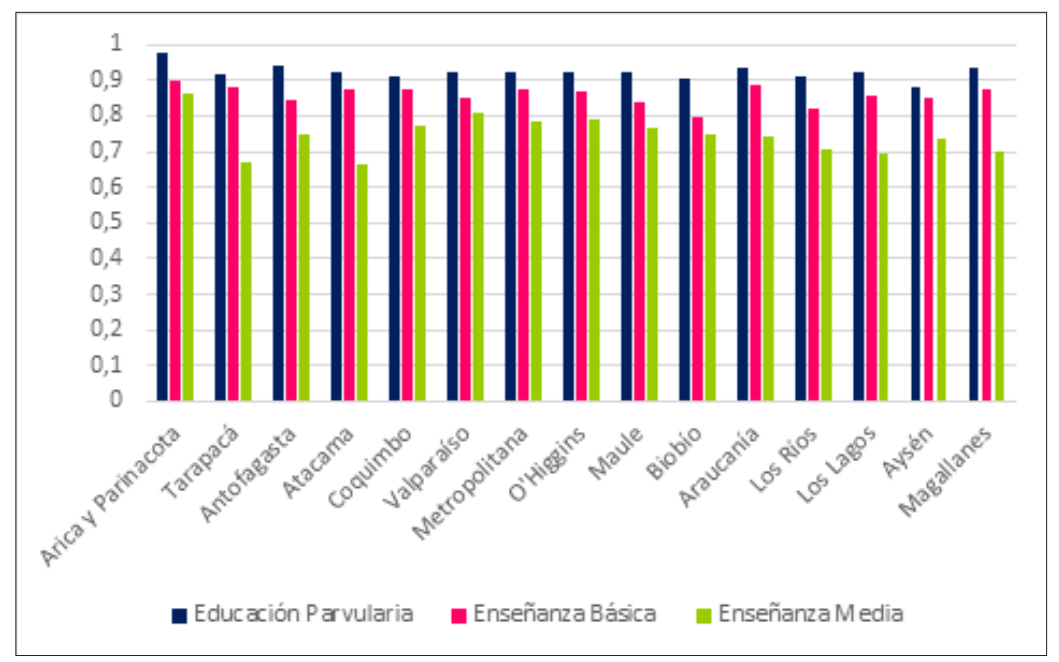

Gráfico 4. Tasas de idoneidad disciplinar según región y nivel de enseñanza.

Fuente: Elaboración propia a partir de la Base de Idoneidad Docente 2016, Mineduc.

Según ruralidad, tal como señala la teoría (Galvis y Bonilla, 2011; Rivero, 2015; Zhou, 2012), se observa una desigualdad que perjudica significativamente al sector rural versus urbano, considerando enseñanza básica ( $84 \%$ versus $75 \%$ ) y media ( $81 \%$ versus 75\%). En enseñanza media (Gráfico 5), las asignaturas con mayores diferencias según ruralidad fueron Ciencias Naturales (61\% versus $45 \%$ ), Biología (67\% versus 51\%), Física (53\% versus 22\%), Química (62\% versus 41\%) y Matemática (81\% versus 73\%). Una tendencia distinta se observó en educación de párvulos, ya que los establecimientos en zonas rurales tienen un índice de idoneidad disciplinar mayor (93\% versus 92\%). 


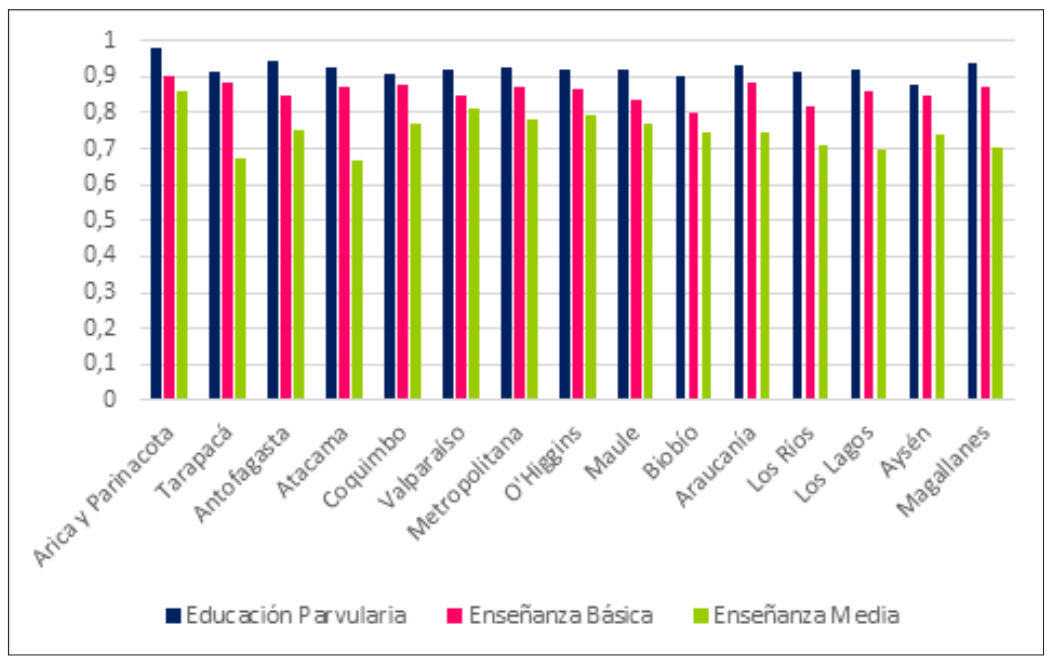

Gráfico 5. Tasas de idoneidad disciplinar en enseñanza media según ramo y ruralidad. Fuente: Elaboración propia a partir de la Base de Idoneidad Docente 2016, Mineduc.

Haciendo un zoom a la Región Metropolitana, y georreferenciando las tasas promedio de idoneidad disciplinar comunal, se aprecian altos niveles de idoneidad en educación básica en sectores rurales, pues prácticamente todas las comunas registran más de un $80 \%$ de horas idóneas. Bajo este porcentaje se encuentran solamente El Monte, Calera de Tango, San Bernardo y Conchalí (ver Mapa 1), mientras Alhué presenta la mayor idoneidad. En el caso de educación media, se observa un patrón centrífugo, donde a mayor distancia del centro de la región se aprecian menores tasas de idoneidad comunal. 


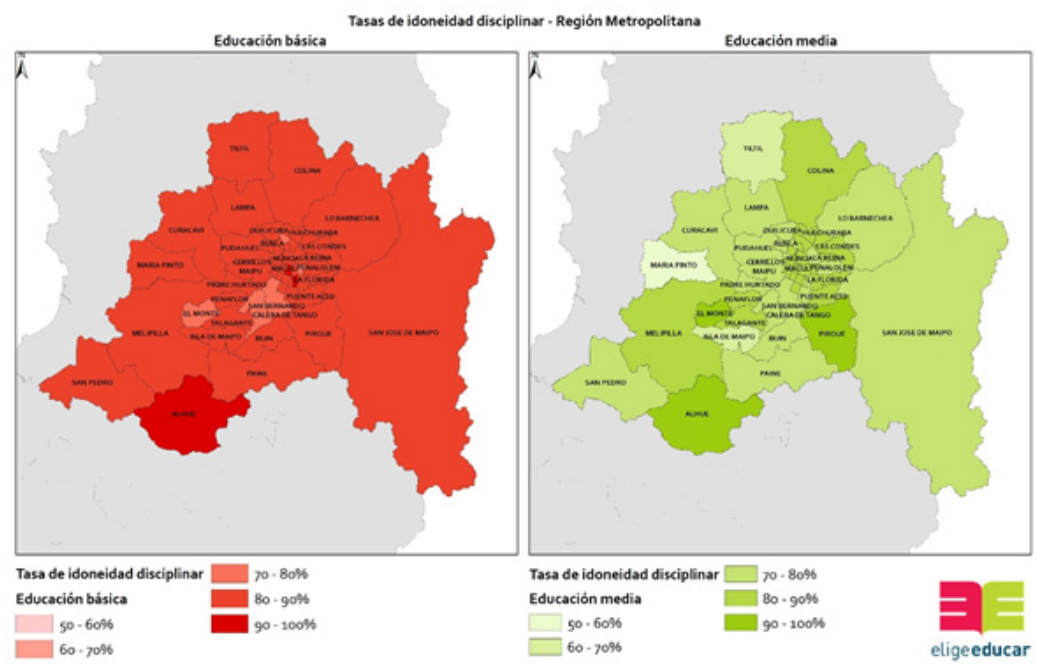

Mapa 1. Tasas de idoneidad disciplinar en enseñanza básica y media, según comuna. Región Metropolitana.

Fuente: Elaboración propia a partir de Base de Idoneidad Docente 2016, y Directorio de Establecimientos, Mineduc.

\subsubsection{Idoneidad disciplinar docente según características de los establecimientos}

Las correlaciones simples entre el índice de idoneidad disciplinar y algunas variables características de los establecimientos muestran resultados interesantes, incluso cuando no proporcionen información causal. Las variables consideradas incluyen GSE, nivel de vulnerabilidad, porcentaje de alumnos prioritarios, retención docente $^{20}$ y tamaño promedio de los cursos. Las Tablas 5,6 y 7 registran los resultados.

20 Esta no se encuentra directamente disponible, por lo que fue necesario construirla (véase en Medeiros et al., 2017). 
72 IDONEIDAD DISCIPLINAR DE LOS PROFESORES Y MERCADO DE HORAS DOCENTES - M. Medeiros, C. Gómez, M. Sánchez, V. Orrego

Tabla 5

Correlaciones entre idoneidad disciplinar y variables descriptivas del establecimiento en educación de párvulos

\begin{tabular}{lc}
\hline Variables & $\begin{array}{c}\text { Correlación con idoneidad } \\
\text { en nivel parvulario }\end{array}$ \\
\hline $\begin{array}{l}\text { Nivel de vulnerabilidad del } \\
\text { establecimiento }\end{array}$ & $-0,353^{* *}$ \\
$\begin{array}{l}\text { Porcentaje de alumnos prioritarios en el } \\
\text { nivel parvulario }\end{array}$ & $-0,158^{* *}$ \\
GSE del establecimiento & $0,350^{* *}$ \\
$\begin{array}{l}\text { Tamaño promedio del curso en el nivel } \\
\text { parvulario }\end{array}$ & $0,234^{* *}$ \\
Retención docente del establecimiento & $0,115^{* *}$ \\
\hline
\end{tabular}

Nota: *con significancia al 90\%; **con significancia al 95\%; y***con significancia al 99\%.

Fuente: Elaboración propia a partir de Base de Idoneidad Docente 2016, Mineduc.

Tabla 6

Correlaciones entre idoneidad disciplinar y variables descriptivas del establecimiento en enseñanza básica

\begin{tabular}{lc}
\hline Variables & $\begin{array}{c}\text { Correlación con idoneidad } \\
\text { en enseñanza básica }\end{array}$ \\
\hline $\begin{array}{l}\text { Nivel de vulnerabilidad del } \\
\text { establecimiento }\end{array}$ & $-0,118^{* *}$ \\
$\begin{array}{l}\text { Porcentaje de alumnos prioritarios en el } \\
\text { establecimiento }\end{array}$ & $-0,100^{* *}$ \\
$\begin{array}{l}\text { GSE del establecimiento } \\
\text { Tamaño promedio del curso en enseñanza } \\
\text { básica }\end{array}$ & $0,150^{* *}$ \\
$\begin{array}{l}\text { Retención docente del establecimiento } \\
\text { Proporción de alumnos dentro un curso } \\
\text { con aprendizaje insuficiente en al menos } \\
\text { una prueba Simce ( } 4^{\circ} \text { año básico) }\end{array}$ & $0,187^{* *}$ \\
$\begin{array}{l}\text { Proporción de alumnos dentro un curso } \\
\text { con aprendizaje insuficiente en al menos } \\
\text { una prueba Simce ( } 8^{\circ} \text { año básico) }\end{array}$ & $0,0811^{* * *}$ \\
\hline
\end{tabular}

Nota: *con significancia al 90\%; **con significancia al 95\%; y ***con significancia al 99\%. Fuente: Elaboración propia a partir de Base de Idoneidad Docente 2016, Mineduc. 


\section{Tabla 7}

Correlaciones entre idoneidad disciplinar y variables descriptivas del establecimiento en enseñanza media

\begin{tabular}{lc}
\hline Variables & $\begin{array}{c}\text { Correlación con idoneidad en enseñanza } \\
\text { media }\end{array}$ \\
\hline $\begin{array}{l}\text { Nivel de vulnerabilidad del } \\
\text { establecimiento }\end{array}$ & $-0,141^{* *}$ \\
$\begin{array}{l}\text { Porcentaje de alumnos prioritarios en el } \\
\text { establecimiento }\end{array}$ & $-0,122^{* *}$ \\
$\begin{array}{l}\text { GSE del establecimiento } \\
\text { Tamaño promedio del curso en } \\
\text { enseñanza básica }\end{array}$ & $0,150^{* *}$ \\
$\begin{array}{l}\text { Retención docente del establecimiento } \\
\text { Proporción de alumnos dentro un curso } \\
\text { con aprendizaje insuficiente en al menos } \\
\text { una prueba Simce (2 } 2^{\circ} \text { año medio) }\end{array}$ & $0,170^{* *}$ \\
\hline
\end{tabular}

Nota: *con significancia al 90\%; ** con significancia al 95\%; y *** con significancia al $99 \%$.

Fuente: Elaboración propia a partir de Base de Idoneidad Docente 2016, Mineduc.

Los resultados evidencian la asociación reportada por la literatura entre idoneidad disciplinar y GSE (Clotfelter et al., 2007; Jerald \& Ingersoll, 2002; Lu et al., 2007; Rivero, 2015; Schultz, 2014; Zhou, 2012) y desde esa perspectiva son intuitivos en sentido y significancia, sin embargo, no poseen la magnitud esperada. Teniendo en cuenta esto, se puede apreciar que mientras mayor es el nivel de vulnerabilidad, más alta es la proporción de estudiantes prioritarios y más bajo el grupo socioeconómico, los establecimientos presentan una menor tasa de idoneidad disciplinar docente para los tres niveles de enseñanza, lo que puede deberse a su dificultad para atraer y contratar docentes idóneos, como plantea Ee-gyeong (2011).

Junto con lo anterior, se observa que aquellos que tienen mayor retención docente también presentan una mayor tasa de idoneidad disciplinar, lo que según Ingersoll (1998) es lógico, pues debiera esperarse que a medida que los docentes dediquen sus horas aula a asignaturas fuera de su especialidad, aumente la probabilidad de rotación y deserción. 
En relación con el Simce, se encontró una correlación significativa y de magnitud mayor al resto entre idoneidad en enseñanza media y proporción de alumnos con aprendizaje insuficiente en, al menos, una prueba Simce en segundo año medio (Tabla 3), lo que genera un nuevo posible foco de investigación acerca de la relación entre idoneidad y aprendizaje.

Finalmente, se detectó que los establecimientos con cursos de mayor tamaño también mostraban un nivel de idoneidad disciplinar mayor. Generalmente los que se ubican en zonas con mayor densidad poblacional, como las zonas urbanas, tienen más facilidad para encontrar al docente que se requiere, señala Ee-gyeong (2011).

\subsubsection{Mercado de horas docentes para 2016}

El total de cargos docentes ${ }^{21}$ a estudiar que cumplían con los criterios antes señalados fue de $191.435^{22}$. El análisis por horas de contrato mostró que solo el 22,73\% trabajaba 44 o más horas semanales. Esto evidencia que existen docentes en el sistema que, considerando posibles escenarios, teóricamente podrían aumentar sus horas de contrato. Del total de cargos docentes, el 44\% trabajaba en establecimientos de administración municipal; mientras que el 44,7\% lo hacía en colegios particular subvencionados; y el 11,11\% en particulares pagados. Por último, es importante recalcar que se presentaron diferencias por género y región. Respecto del género, $72 \%$ de los cargos correspondió a mujeres y $28 \%$ a hombres. En términos de distribución regional, existe concentración en la RM, la cual acumula 34\% de los cargos docentes, seguida por Biobío y Valparaíso. Las regiones extremas presentan menor cantidad de cargas docentes y solo el 13\% se desempeña en zonas rurales.

21 El docente puede hacer clases en más de un establecimiento, por lo tanto, la unidad de muestra a considerar en este estudio es el "cargo docente" (Sepúlveda, 2015), el cual se define como el acuerdo entre el establecimiento y este, que implica que este último trabaja cierto número de horas haciendo clases en ese colegio. Un mismo docente puede tener más de un cargo si trabaja en más de un establecimiento.

22 El total de cargos docentes de aula (191.435) corresponde a 180.277 docentes, de los cuales el $95 \%$ trabaja en un solo establecimiento, y cerca del 5\% restante en dos o más establecimientos. 
En relación con la demanda del año 2016, de los 101.510 cursos en el país, en promedio el 68\% tiene jornada escolar completa. Respecto de la distribución de los alumnos, las regiones que poseen mayor matrícula son la Región Metropolitana (37\%), Biobío (12\%) y Valparaíso (10\%).

Como se reporta en la Tabla 8, para el año 2016 se obtuvo como resultado que para los tres niveles de enseñanza, existía superávit de horas docentes totales. Para educación parvularia, ello implicó un $8 \%$ de excedente de horas totales y $2 \%$ para enseñanza básica. Cabe destacar que en ambos niveles existen regiones con déficit de horas: en educación de párvulos las regiones con déficit fueron Antofagasta, O'Higgins y La Araucanía, mientras que para enseñanza básica fueron La Araucanía, Los Lagos, Metropolitana y Los Ríos.

En enseñanza media, este excedente alcanzó un $64,5 \%$ en Lenguaje y comunicación; 58,62\% en Matemáticas; 42,86\% en Ciencias; y 54,66\% en Historia.

Tabla 8

Mercado de horas docentes al año 2016 sin considerar idoneidad disciplinar

\begin{tabular}{l|cccc}
\hline & Oferta & Demanda & Resultado & $\begin{array}{c}\text { Resultado/ } \\
\text { oferta }\end{array}$ \\
\hline Educación de párvulos & 1.612 .167 & 1.482 .546 & 129.621 & $8,04 \%$ \\
Enseñanza básica & 7.488 .999 & 7.326 .134 & 162.865 & $2,17 \%$ \\
$\begin{array}{l}\text { Enseñanza media: Lenguaje y } \\
\text { comunicación }\end{array}$ & 693.457 & 253.128 & 440.329 & $63,50 \%$ \\
$\begin{array}{l}\text { Enseñanza media: Ciencias } \\
\text { Enseñanza media: Historia y }\end{array}$ & 568.409 & 324.792 & 243.617 & $42,86 \%$ \\
geografía & 477.522 & 216.528 & 260.994 & $54,66 \%$ \\
$\begin{array}{l}\text { Enseñanza media: } \\
\text { Matemáticas }\end{array}$ & 671.932 & 278.074 & 393.858 & $58,62 \%$ \\
\hline Total & 11.512 .485 & 9.881 .202 & 1.631 .283 & $14,17 \%$ \\
\hline
\end{tabular}

Fuente: Elaboración propia a partir de Base Idoneidad Docente y Matrícula, Mineduc (varios años). 


\subsubsection{Proyección de mercado de horas docentes para 2017-2031}

En el Gráfico 6 se observa que, para la educación de párvulos, en el escenario 1 (más pesimista), el déficit al año 2031 alcanzaría un $67,3 \%$ sobre las horas ofrecidas, en cambio, si aumentara la atracción hacia la carrera de Educación de Párvulos a partir de la PND, este déficit disminuiría a 58,9\%. Así mismo, este se reduciría a 1,8\% cuando los educadores aumenten sus horas de contrato, distribuyéndolas según las necesidades de su región. Las regiones que presentarían mayores problemas son Los Ríos, Los Lagos y Libertador Bernardo O'Higgins. Se debe tener en cuenta que la demanda está subestimada en esta proyección, debido a que no se incluye la demanda de educadores de párvulos en jardines infantiles y salas cuna. Por lo tanto, si se consideraran estos niveles de demanda por educadores de párvulos, el déficit sería aún mayor en todos los escenarios presentados.

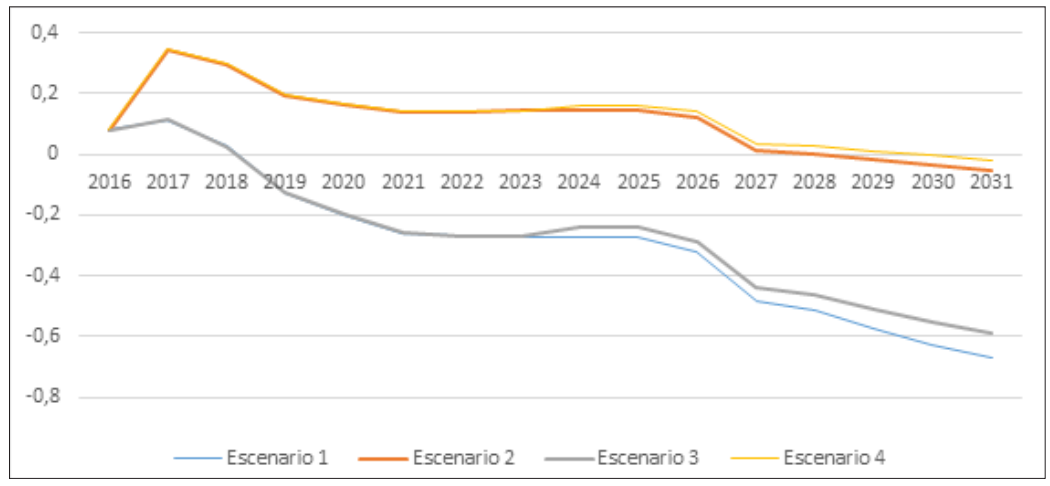

Gráfico 6. Déficit o (superávit) sobre oferta mensual de horas docentes en educación de párvulos.

Fuente: Elaboración propia a partir de Base Idoneidad Docente, Matrícula y Rendimiento por alumno, Mineduc. Matrícula y Seleccionados, SIES. Estadísticas por carrera, Mi Futuro e Índices Matrícula, CNED (varios años).

Del mismo modo, para el 2031 en enseñanza básica existiría un déficit mayor al 100\% en todos los escenarios posibles. Esto ocurre, en parte, porque la disminución de la matrícula no estaría siendo contrarrestada por el aumento en el interés por esta Pedagogía, ni por las horas de contrato que les falta a los docentes del sistema para completar el contrato a horario completo dado que, en promedio, en su contrato tienen un número de horas más altas que el resto de 
los docentes. Otra razón por la cual el déficit en este nivel de enseñanza sería tan alto se debe a que el estado del mercado al año 2016 fue casi de equilibrio, a diferencia del resto de los niveles que reportan un superávit en ese año. Esto se debe a que la demanda por estos docentes fue mayor porque imparten la mayoría de las asignaturas de su nivel de enseñanza. Mientras que la demanda en horas de un curso de enseñanza media por un docente con especialidad en Matemáticas es de 24 horas cronológicas mensuales, la demanda de básica es 108 horas $^{23} 24$. Sin embargo, en las regiones de Los Ríos, Los Lagos y Libertador Bernardo O'Higgins persiste el déficit, incluso en el mejor de los escenarios, principalmente por la dificultad para contrarrestar la disminución de la proporción de horas lectivas con las horas de contrato faltantes de los docentes para obtener uno a tiempo completo (Gráfico 7). Las regiones más afectadas en este caso fueron la de Tarapacá y Antofagasta.

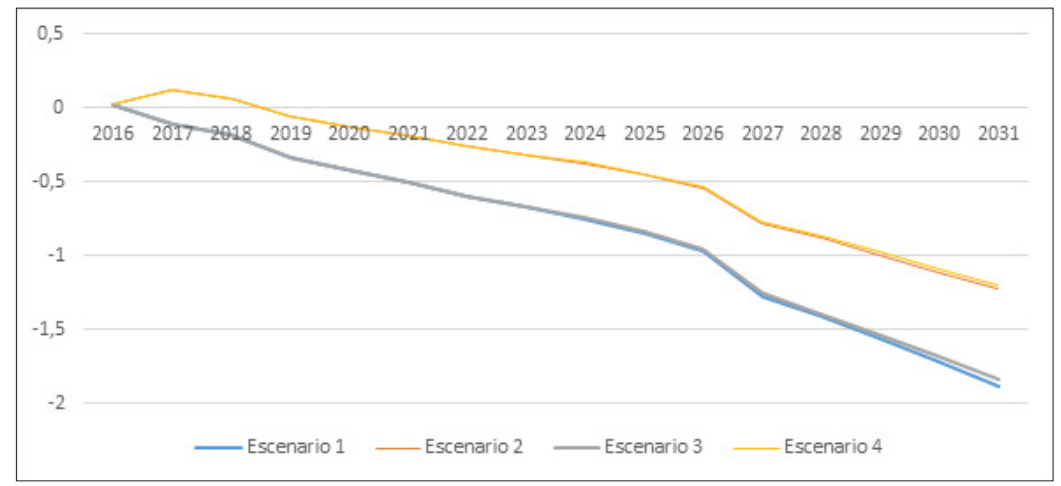

Gráfico 7. Déficit o (superávit) sobre oferta mensual de horas docentes en enseñanza básica.

Fuente: Elaboración propia a partir de Base Idoneidad Docente, Matrícula y Rendimiento por alumno, Mineduc. Matrícula y Seleccionados, SIES. Estadísticas por carrera, Mi Futuro e Índices Matrícula, CNED (varios años).

23 Estos números corresponden a lo que el Mineduc, a través de su Plan de Estudios exige a los establecimientos adscritos al régimen de Jornada Escolar Completa. En el caso de enseñanza básica se incluye Lenguaje, Matemáticas, Historia, Ciencias Naturales, Orientación y Consejo de curso. En cambio, para primero y segundo año medio en la modalidad científico humanista, se deben impartir 24 horas de Matemáticas.

24 Otras de las razones que pueden estar ocasionando el gran déficit en este nivel de enseñanza es la limitación de datos que existe en el reporte del Mineduc en su Base de Idoneidad Docente, en donde los docentes de básica solo pueden reportar dos de las asignaturas que realizan, lo que puede generar una subestimación de la oferta. 
En enseñanza media (ver Gráficos 8, 9, 10 y 11), el caso más crítico es el de Ciencias (que incluye Ciencias Naturales, Física, Biología y Química) que presentaría déficit en todas las regiones en el escenario más negativo (escenario 1), y en el optimista (escenario 4), solo las regiones del Maule, Valparaíso y Biobío presentan superávit. La región más crítica fue Atacama que mostró un déficit de 363\% en el escenario 4. A diferencia de enseñanza básica, la principal razón del déficit de docentes en Ciencias fue la escasa oferta que existía al año 2016, y la oferta que se proyectó. Es importante, en esta área en particular, poner el foco en atracción a la carrera de Pedagogía.

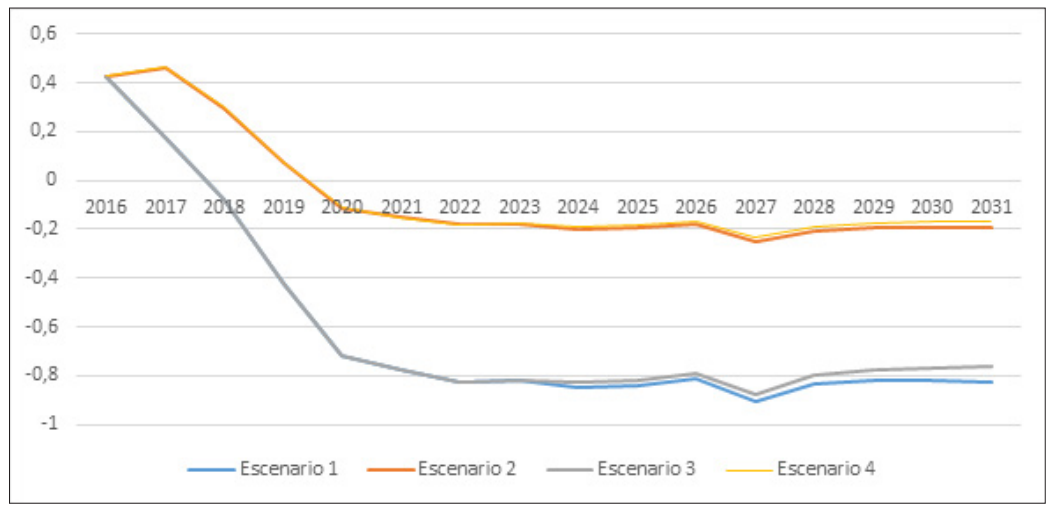

Gráfico 8. Déficit o (superávit) sobre oferta mensual de horas docentes de ciencias en enseñanza media.

Fuente: Elaboración propia a partir de Base Idoneidad Docente, Matrícula y Rendimiento por alumno, Mineduc. Matrícula y Seleccionados, SIES. Estadísticas por carrera, Mi Futuro e Índices Matrícula, CNED (varios años).

El caso de Historia, Lenguaje y Matemática es menos crítico, sin embargo, en las tres asignaturas existen regiones que presentarán déficit al año 2031, incluso en el escenario más positivo, por ejemplo, las regiones de Los Ríos y Atacama tendrán un déficit en Historia de $312,5 \%$ y 98,2\%, respectivamente; Antofagasta y Atacama tendrán un déficit de 75\% y 187,4\% en Lenguaje y comunicación, respectivamente; y Atacama presentará un déficit de 138,2\% en Matemáticas. 


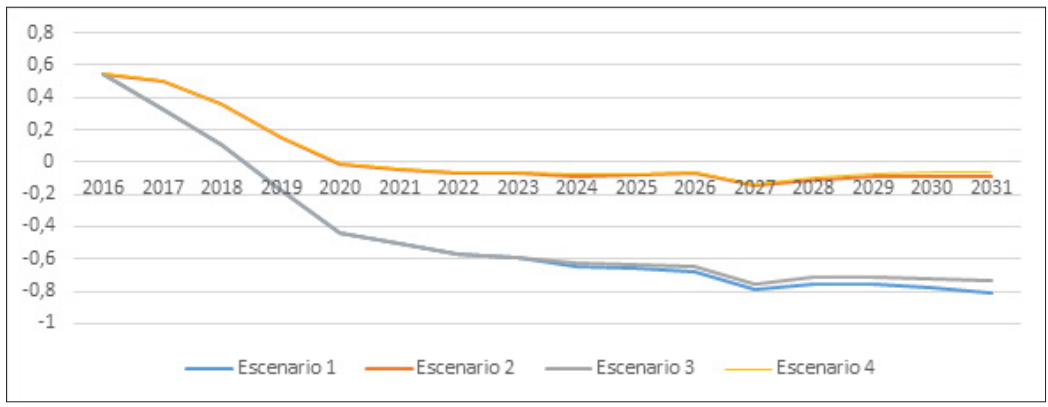

Gráfico 9. Déficit o (superávit) sobre oferta mensual de horas docentes de Historia en enseñanza media.

Fuente: Elaboración propia a partir de Base Idoneidad Docente, Matrícula y Rendimiento por alumno, Mineduc. Matrícula y Seleccionados, SIES. Estadísticas por carrera, Mi Futuro e Índices Matrícula, CNED (varios años).

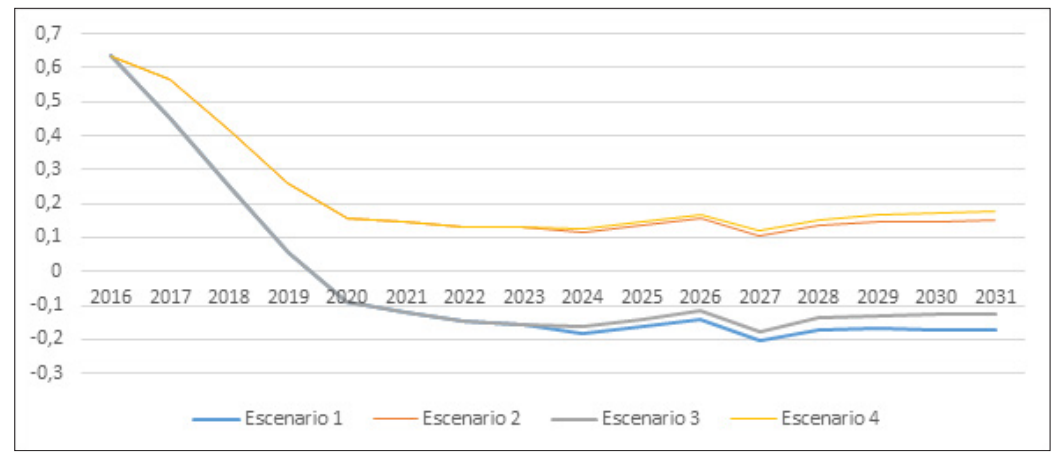

Gráfico 10. Déficit o (superávit) sobre oferta mensual de horas docentes de Lenguaje y comunicación en enseñanza media.

Fuente: Elaboración propia a partir de Base Idoneidad Docente, Matrícula y Rendimiento por alumno, Mineduc. Matrícula y Seleccionados, SIES. Estadísticas por carrera, Mi Futuro e Índices Matrícula, CNED (varios años). 


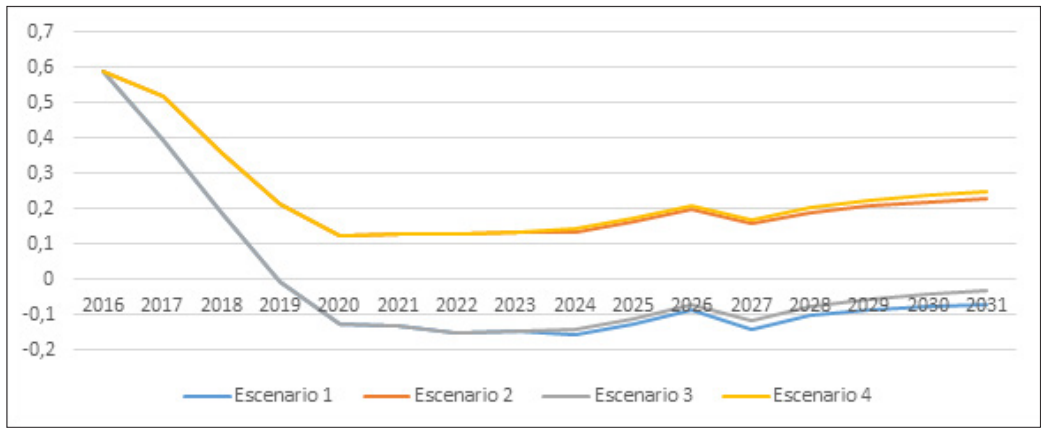

Gráfico 11. Déficit o (superávit) sobre oferta mensual de horas docentes de Matemáticas en enseñanza media.

Fuente: Elaboración propia a partir de Base Idoneidad Docente, Matrícula y Rendimiento por alumno, Mineduc. Matrícula y Seleccionados, SIES. Estadísticas por carrera, Mi Futuro e Índices Matrícula, CNED (varios años).

En conclusión, teniendo en cuenta que existen múltiples escenarios posibles, dadas las modificaciones al sistema por la PND, si se mantienen las condiciones actuales, entonces se generará un déficit de horas de clase en todas las regiones y niveles de enseñanza. En cambio, en un escenario más positivo, en donde hay reacciones a partir de la PND, el déficit se vería contrarrestado en parte, aunque en algunas ocasiones no lo suficiente, para volver al estado inicial del año 2016. Por ello, se hace fundamental acompañar la implementación de la PND con políticas fuertes de atracción a las carreras de educación.

Finalmente, se proyectó considerando como estado inicial de 2016 solo las horas de clase realizadas por docentes con la especialidad respectiva, es decir, se contemplaron solo las horas aula idóneas, manteniendo constante todo el resto de los supuestos y estimaciones. Como se reporta en las Tablas 9 y 10, al poner atención a la idoneidad disciplinar, los resultados para el año 2016 son más críticos. Para enseñanza básica, se comienza con un déficit de 13,6\% el 2016, por lo que el escenario 1 al 2031 reportaría un déficit de $443,6 \%$, aún mayor que la proyección sin considerar idoneidad. Para las asignaturas de Ciencias, Historia, Lenguaje y Matemáticas en enseñanza media ocurre algo parecido, en donde el déficit reportado a 2031 en la proyección con idoneidad es mayor a aquel sin idoneidad (casi el doble), como es el caso de Ciencias. 
Tabla 9

Mercado de horas docentes al 2016 considerando la idoneidad disciplinar de los docentes

\begin{tabular}{lrrrr}
\hline & Oferta & Demanda & Resultado & $\begin{array}{c}\text { Resultado/ } \\
\text { Oferta }\end{array}$ \\
\hline Educación de párvulos & 1.485 .737 & 1.482 .546 & 3.191 & $0,20 \%$ \\
Enseñanza básica & 6.447 .153 & 7.326 .134 & -878.981 & $-13,60 \%$ \\
$\begin{array}{l}\text { Enseñanza media: Lenguaje y } \\
\text { comunicación }\end{array}$ & 611.168 & 253.128 & 358.040 & $58,60 \%$ \\
Enseñanza media: Ciencias & 339.787 & 324.792 & 14.995 & $4,40 \%$ \\
$\begin{array}{l}\text { Enseñanza media: Historia y } \\
\text { geografía }\end{array}$ & 444.494 & 216.528 & 227.966 & $51,30 \%$ \\
Enseñanza media: Matemáticas & 543.807 & 278.074 & 265.733 & $48,90 \%$ \\
\hline Total & 9.872 .146 & 9.881 .202 & -9.056 & $-0,10 \%$ \\
\hline
\end{tabular}

Fuente: Elaboración propia a partir de Base Idoneidad Docente, Matrícula y Rendimiento por alumno, Mineduc. Matrícula y Seleccionados, SIES. Estadísticas por carrera, Mi Futuro e Índices Matrícula, CNED (varios años). 


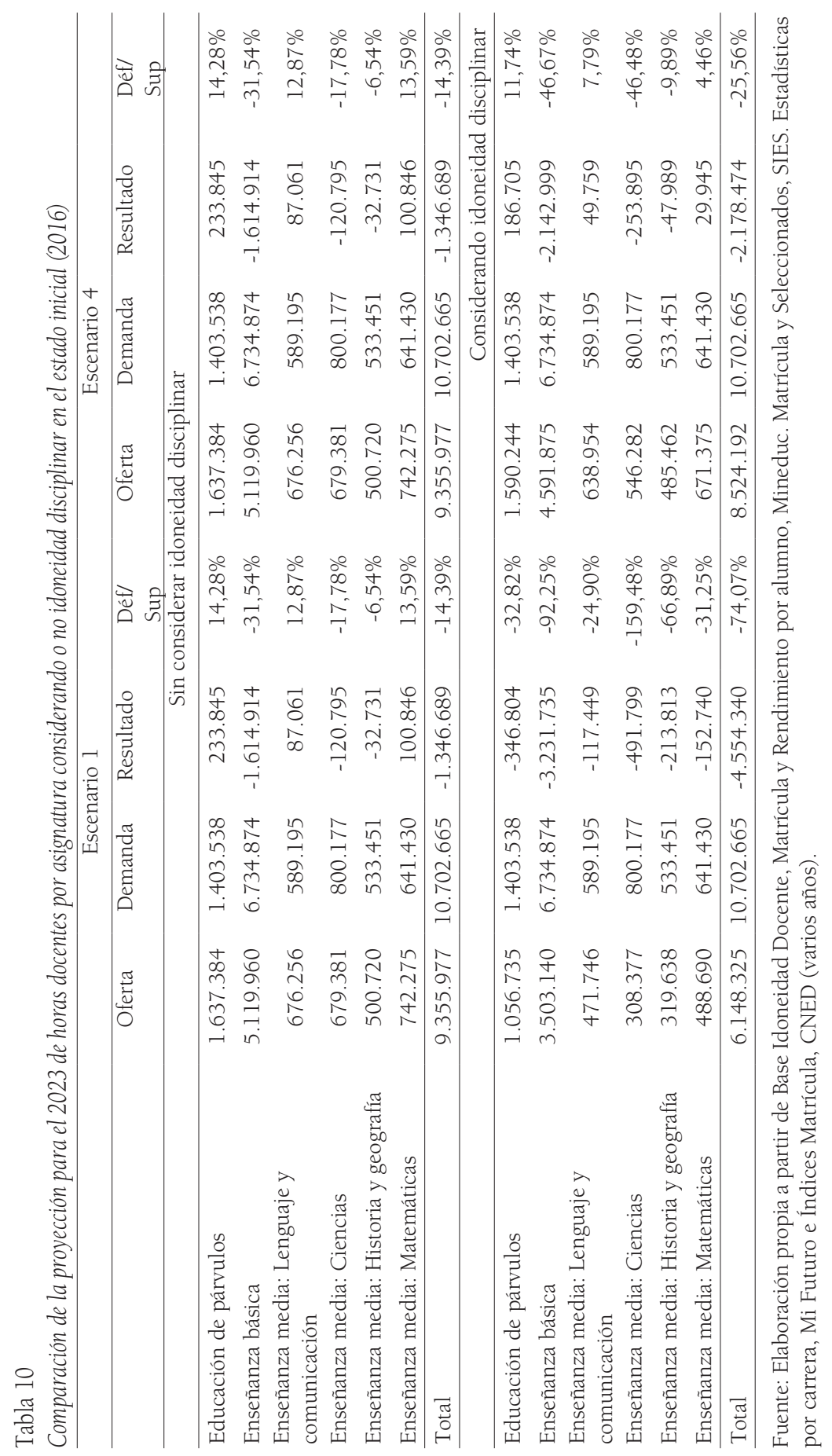




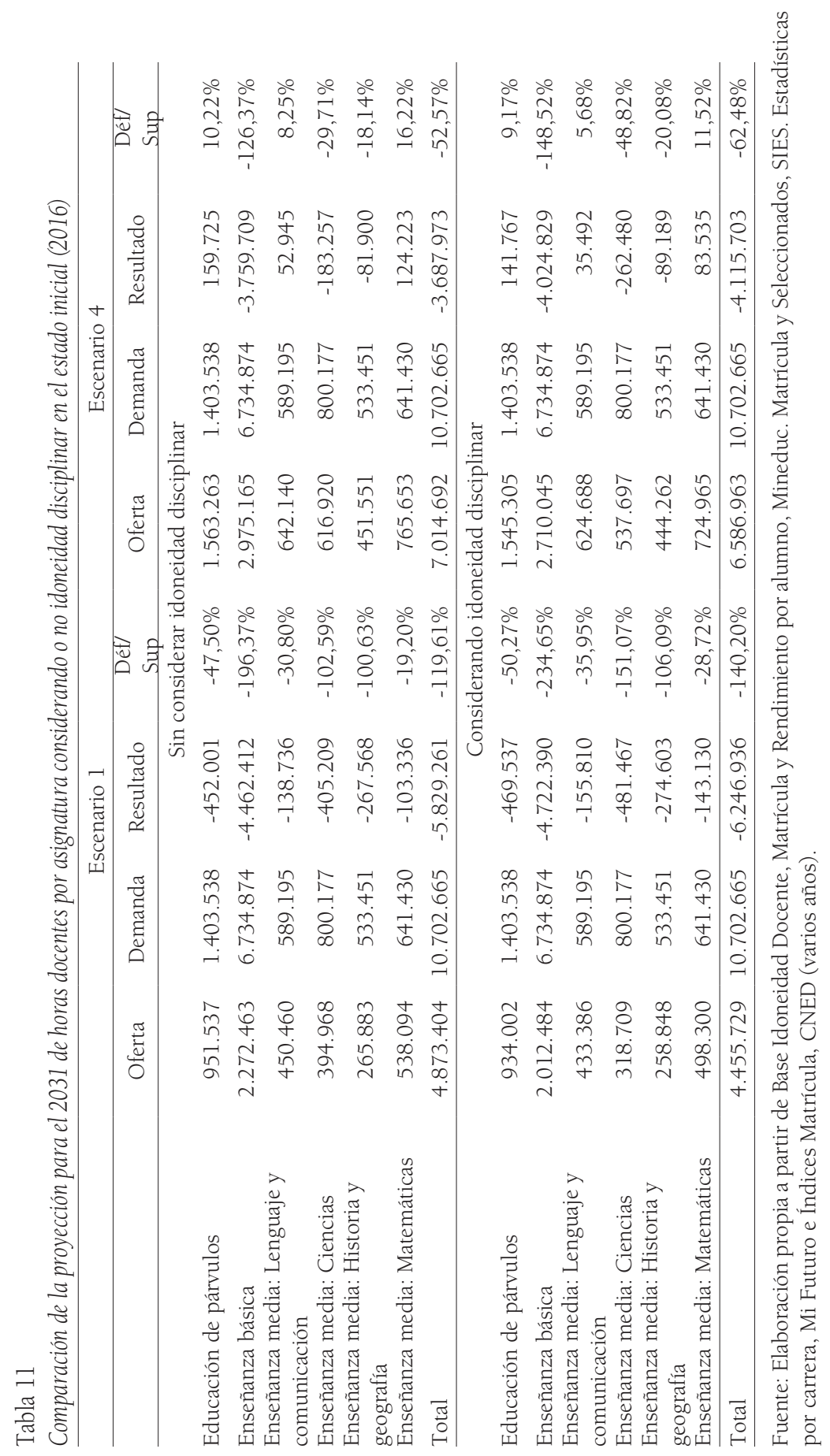




\subsection{Análisis cualitativo}

\subsubsection{Idoneidad docente}

Los directores entrevistados ofrecen un discurso moderado frente a la idoneidad disciplinar, declarando que la mayor parte de su planta tendría la especialización respectiva, excepto algunos casos habilitados por Mineduc. Sin embargo, son capaces de percibir cierta abundancia y déficit, particularmente durante la búsqueda de nuevos profesionales. Docentes para asignaturas como Historia y geografía, Educación física e Inglés serían mucho más sencillos de contratar debido a la oferta existente que profesionales en Física, Biología y Tecnología, situación que coincide con algunos de los datos reportados en la sección cuantitativa. A pesar de esto, reiteran, nunca un curso queda sin profesor, pues se recurre a las habilitaciones ministeriales de egresados de Pedagogía en proceso de titulación y/o profesionales de disciplinas afines. Llama la atención que estas ocurran especialmente en Matemáticas y Física en enseñanza media y en las especializaciones técnicas en la educación técnico-profesional. De esa manera, más que estar frente a vacantes que no se pueden llenar (vacancy rates), este fenómeno parece invisibilizar una escasez escondida (hidden shortages), siguiendo los términos empleados por Jackson (2009) y Santiago (2002).

Aunque en ningún caso los entrevistados indican que las habilitaciones son la primera opción al momento de la contratación, estas se comprenden como una necesidad del funcionamiento escolar. Tampoco supondrían desmedro de los aprendizajes, amenazas al cumplimiento del currículo o de las horas mínimas requeridas por los planes de estudio. Por el contrario, los problemas de calidad referidos a la escasez escondida residirían, para los directores, más bien en los profesores titulados contratados como reemplazantes, debido a la premura de los tiempos que imposibilita procesos de selección satisfactorios. Uno de los entrevistados comentó al respecto:

Nada te asegura que, por tener un título de Pedagogía, vas a enseñar bien, ¿ya? Porque puede venir una persona que realmente no tiene el título y va a lograr los aprendizajes esperados o lo que 
tú quieres con los objetivos de la clase mejor que un profe que está preparado para ello (Entrevista 4, Director Región de Valparaíso).

\subsubsection{Mercado docente}

En términos de la oferta y demanda, en general, se aprecia que las opiniones de los entrevistados tienden a reconocer en cierta medida el incumplimiento de las horas mínimas exigidas por los planes de estudio. No obstante, este fenómeno no estaría afectado por la falta de profesionales con la especializada respectiva, sino más bien por las licencias médicas, permisos administrativos y, en menor medida, paralizaciones de estudiantes y trabajadores, detención por reflexión y evaluaciones provenientes del nivel central.

Definidos como imprevistos, estos se asumen como parte inherente del sistema laboral y educacional y su grado de influencia dependería de la administración de los recursos financieros que permiten contratar profesionales de apoyo; siendo especialmente significativo su alto número o su participación estable por medio de contrataciones anuales o en jornada completa. Ahora bien, lo cierto es que los imprevistos suelen favorecer el out-of-field teaching, en tanto la clase es cubierta por profesionales de apoyo que no poseen la especialización requerida específicamente, paradocentes o profesionales de otras asignaturas. En el caso de los últimos, además, puede significar la disminución de las horas no electivas. Uno de los entrevistados señala al respecto:

Esto es un efecto dominó. El no tener un profesor que esté cubriendo un curso, significa que otro profesor, de otra asignatura, tiene que reforzar y estar trabajando ahí a pesar de que no le corresponde. Entonces ahí, en establecimientos como este, se trabaja mucho con la solidaridad entre pares (Entrevista 4, Director Región de Valparaíso).

Otro elemento interesante en este punto es el superávit y déficit de horas pedagógicas. Algunos elementos señalados por los entrevistados permiten comprender parte de estos fenómenos. El superávit podría relacionarse con la necesidad de refuerzo académico de ciertas asignaturas, sea o no con vistas al Simce, según 
el diagnóstico particular de cada establecimiento. En Historia, por ejemplo, dos de los entrevistados suman una hora al calendario para realizar el curso de Formación cívica, en una decisión que se adelantó al debate educacional respectivo. El ejercicio más común es usar las horas de libre elección, seguido por la disminución de horas de asignaturas percibidas como menos relevantes, reducción de los cursos de taller o su desplazamiento fuera de la jornada regular y, por último, la recuperación de clases o realización de reforzamientos fuera del horario escolar.

\subsubsection{Contratación de profesores}

Respecto de los procesos de búsqueda y selección, tal como muestra Rivero, M. (2015), existen diferencias entre los establecimientos según idoneidad disciplinar. En primer lugar, en la presente investigación aparecieron tres estrategias principales para buscar profesionales: i) contactos personales; ii) convocatorias abiertas empleando redes sociales, diarios y corporación municipal y iii) prácticas profesionales. Los establecimientos con idoneidad baja tienden a las dos primeras; mientras aquellos con idoneidad alta se transforman en centros de práctica profesional, hecho que les permite no solo conocer previamente a los mejores egresados, sino también incidir en su formación inicial y, posterior inserción laboral. Esta diferencia retrata la existencia de dinámicas organizacionales más efectivas entre los establecimientos con mayores tasas de idoneidad para captar a los docentes más calificados.

Durante este proceso, los establecimientos con alta idoneidad tienden a recibir mayor número de postulaciones y pueden aplicar criterios estrictos de selección, incluyendo predilección por ciertas universidades. Debido al menor número de currículums que reciben aquellos establecimientos con idoneidad baja, dichos criterios se vuelven más laxos. Entre los criterios de selección aparecen fuertemente mencionados aquellos referidos a las habilidades blandas, particularmente al trabajo en equipo y la preocupación por el aprendizaje de los estudiantes. En las escuelas situadas en contextos de alta vulnerabilidad, las habilidades toman mayor centralidad, pudiendo incluso dejar como criterio secundario el conocimiento disciplinar. Al respecto, uno de los directores señala: 
Porque acá a veces una falencia técnica, se subsana con una habilidad social. ¿Ya? Porque puedes tener un doctor en Matemáticas, en Lenguaje y si no tiene habilidad social, no te va a servir dentro de este establecimiento (Entrevista 4, Región de Valparaíso).

El principal problema identificado, además del menor número de postulantes en ciertas asignaturas previamente indicado, es externo a la búsqueda de profesionales y reside en las horas de contrato ofrecidas: siempre será más complejo buscar a un profesional a quien no se le puede ofrecer contrato por jornada completa. Ambas dificultades afectan especialmente a la región de Atacama y, según la opinión de los entrevistados, guarda estrecha relación con el menor número de profesionales formados en la región, la escasez de especializaciones y la baja calidad de los programas locales. Estos hallazgos concuerdan con lo detectado por el análisis cuantitativo, que consignaba que la zona norte presenta mayor déficit de horas docentes.

A modo de conclusión de la fase cualitativa, se observó que los directores tendían a presentar un discurso más moderado frente a la idoneidad docente de lo que reflejan los datos cuantitativos. No obstante, al profundizar en ello aparecen no solo los problemas al momento de la búsqueda de profesionales, sino también el fenómeno de out-of-field teaching, siendo el invierno la época más crítica debido al alto número de licencias médicas cursadas en dicho periodo. Lamentablemente, no se encontró evidencia del efecto que tiene la baja idoneidad disciplinar, especialmente en contextos de alta vulnerabilidad social. Es cierto que las habilidades blandas son centrales dentro del aula, pero estas deben considerarse como un elemento más de la ecuación y no pueden sustituir el contenido disciplinar.

\section{Conclusiones y recomendaciones}

El presente estudio da cuenta de la relevancia de estudiar la idoneidad disciplinar de la planta docente, el mercado laboral de los profesores en Chile, y los cambios que la nueva PND impondrá al sistema 
educativo. Tal como ha emergido en la literatura internacional analizada (Ee-gyeong, 2011; Ingersoll,1998; Lu et al., 2007), la baja idoneidad disciplinar tiende a concentrarse en ciertos contextos y disciplinas. Analizando la distribución según dependencia administrativa, los establecimientos municipales están claramente más afectados, especialmente en Ciencias Naturales, Tecnología y Lenguaje. En cambio, los colegios particulares pagados presentan problemas únicamente en Inglés y poseen, contrario a la tendencia general, idoneidad más alta en Matemática. Siguiendo el tono de otras investigaciones latinoamericanas (Castro \& Esposito, 2017; Galvis y Bonilla, 2011; Rivero, 2015), se releva la inequidad en la distribución geográfica con problemas específicos en las regiones de Aysén, Carlos Ibáñez del Campo y Biobío en educación inicial; en Arica, Maule y Biobío respecto de la educación básica; y Atacama en educación media.

En relación con el mercado de horas docentes, en 2016 se observa una tendencia al superávit en la mayoría de las asignaturas y niveles, tal como reportan De Hek et al. (2017), Sánchez et al. (2013) y Sepúlveda (2015), sumando algunas excepciones críticas, especialmente concentradas en la zona norte del país. Sin embargo, la proyección muestra déficit para todos los niveles de enseñanza y asignaturas tras la implementación de la PND, siempre que no se creen mecanismos para incentivar la atracción hacia la carrera de Pedagogía y sin compensar el aumento de horas no lectivas con más docentes nuevos en el sistema. Incluso en el mejor escenario, hay asignaturas y niveles de enseñanza que presentarán déficit, especialmente en las regiones del norte.

En consonancia con lo anterior, el levantamiento cualitativo evidenció los problemas que presentan los establecimientos para contratar docentes idóneos suficientes para cubrir las horas requeridas por los planes de estudio del Ministerio de Educación. Dos ideas llaman la atención y supondrían líneas de investigación futuras interesantes de profundizar. Por un lado, el vínculo entre idoneidad y absentismo docente, particularmente crítico en los meses de invierno debido al alto número de licencias médicas cursadas. Por otro, están las estrategias utilizadas por los directivos para resolver las dificultades asociadas 
a la idoneidad disciplinar y al mercado docente, concretamente se trata de comprender los efectos que tendrían el reemplazo de ciertas asignaturas y las habilitaciones ministeriales sobre el aprendizaje de los alumnos. Aunque la literatura ha señalado diversos factores involucrados para hablar acerca de la calidad docente, poco se ha dicho en relación con las habilidades blandas asociadas a la enseñanza, un elemento crítico fuertemente reportado por los entrevistados y particularmente relevante en contextos de vulnerabilidad.

Un elemento transversal a los resultados son los patrones de desigualdad en la distribución de los docentes, reportados también por Clotfelter et al. (2007), Jerald y Ingersoll (2002), Rivero (2015), Schultz (2014) y Zhou (2012), entre otros. En otras palabras, se observa una tendencia que articula menor idoneidad disciplinaria a mayor vulnerabilidad, mayor número de estudiantes prioritarios y menor GSE. El tamaño de las escuelas (relacionado con la mayor idoneidad) podría matizar estos resultados y aminorar dichas correlaciones. Asimismo, se observa una asociación entre idoneidad disciplinar y resultados en los aprendizajes (medidos a través de la proporción de alumnos con aprendizajes insuficientes en, al menos, una prueba Simce de $2^{\circ}$ año medio) para el caso de enseñanza media. Un análisis econométrico causal podría estudiar mejor esta dinámica, pues si bien las correlaciones obtenidas no fueron altas, sí fueron significativas y de sentido esperado.

En este contexto, pueden identificarse siete estrategias que podrían contribuir a mejorar la idoneidad disciplinar — especialmente en el nivel de educación media donde contar con especialización es una exigencia por Decreto de Ley-, y disminuir la brecha entre oferta y demanda de docentes proyectada en el país. En general, las recomendaciones se enmarcan en las discusiones internacionales que, según Knoeppel (2007), articulan la distribución docente bajo el principio de equidad horizontal y vertical, es decir, se trata de crear políticas donde todos los colegios cuenten con docentes de calidad, priorizando en aquellos con mayores necesidades.

Respecto de la idoneidad disciplinar, en primer lugar, se propone generar mecanismos que permitan hacer seguimiento a 
las regulaciones impuestas por el Decreto 352 de la Ley 18.956. Esta responsabilidad podría recaer en la Agencia de Calidad de la Educación, incorporando dicho criterio en el monitoreo de la calidad de los establecimientos educacionales. En segundo lugar, se sugiere desarrollar un sistema de incentivos para atraer y retener a los docentes, focalizado en asignaturas, niveles y zonas con mayores dificultades (Castro \& Esposito, 2017; Clotfelter et al., 2007; Galvis y Bonilla, 2011). Una medida en esta línea podría ser la modificación de la Bonificación de Reconocimiento Profesional mención para permitir un mayor reconocimiento en los sectores con mayores déficits presentes y proyectados, o bien, pensar en otros beneficios (como mayores posibilidades de perfeccionamiento) o asignaciones para los docentes que se desempeñen en contextos de baja idoneidad disciplinar. Esta última medida puede, además, acortar las brechas en la calidad de enseñanza, señala Knoeppel (2007).

En un escenario de mayores exigencias e incentivos, es central además desarrollar mecanismos de apoyo directo a los establecimientos educacionales. Por un lado, abocados a los procesos de contratación (Clotfelter et al., 2007; Zhou, 2012), por ejemplo, flexibilizando los recursos SEP para permitir la contratación de profesores en caso de déficit y en casos de mayor vulnerabilidad regional, rural y por GSE y, a propósito de los resultados cualitativos, incentivar la relación entre los establecimientos y las instituciones de educación superior, para que se constituyan en centros de práctica profesional. Por otra parte, se propone diseñar políticas de contratación diferenciadas para establecimientos de menor matrícula, con especial foco en aquellos rurales, unidocentes y multigrado.

Por otro lado, en términos de adelantarse y evitar los déficits proyectados en el mercado docente, se sugiere generar políticas orientadas hacia una mayor atracción de estudiantes a las carreras de educación. El trabajo en conjunto con las facultades de educación de las distintas regiones es transcendental en esta tarea, especialmente en zonas con mayores déficits. Es relevante, además, considerar no solo a los recién egresados de enseñanza media, sino también a otros profesionales con vocación tardía y, por ello, se propone también la reactivación de la Beca Vocación de Profesor tipo III (también llamada 
de "vocación tardía"), ampliándola al financiamiento de programas de formación pedagógica orientados a las especialidades de educación media con mayor déficit y a las carreras de Educación General Básica y Educación de Párvulos. Junto con lo anterior, resultará clave que se haga un esfuerzo comunicacional para socializar los nuevos beneficios y condiciones de la Política Nacional Docente, así como continuar desarrollando campañas que busquen relevar la valoración social de esta profesión, para que la realidad se acerque al escenario más optimista, donde los cambios en selectividad y remuneraciones efectivamente aumenten el interés por estudiar carreras de educación.

Finalmente, es relevante considerar las limitaciones del estudio a la hora de interpretar estos resultados. La limitación más importante corresponde a la existencia de brechas de información que impidieron desarrollar un diagnóstico más preciso, tal como fue señalado en los supuestos del modelo de proyección, y que pueden reflejar diferencias substanciales en los resultados. Por ejemplo, en educación parvularia, el presente análisis sobreestimó la idoneidad y mercado docente en los niveles de transición (NT1-NT2) y, al mismo tiempo, excluyó los niveles de sala cuna y medio menor, dada la ausencia de información acerca de ellos, desarrollados principalmente por JUNJI, Integra y VTF, y bajo una histórica desregulación y desinformación de los establecimientos privados. De esa manera, se hipotetiza que el comportamiento de la idoneidad disciplinar en este nivel podría diferir significativamente de los demás niveles analizados.

Ahora bien, independientemente de esta limitación, el presente estudio ha permitido no solo actualizar las proyecciones del mercado docente - considerando los cambios que se generarán por implementación de la PND—, sino también analizar en profundidad el fenómeno de la idoneidad disciplinar. Los desafíos propuestos son extensos y se enraízan en los lineamientos más amplios que afectan la reforma educacional en Chile, esperando mejorar la profesión docente y, a través de ello, la calidad de la enseñanza que recibe cada estudiante del país. 


\section{Referencias}

Barber, M. y Mourshed, M. (2008). ¿Cómo hicieron los sistemas educativos con mejor desempeño del mundo para alcanzar sus objetivos? Santiago de Chile: CINDE.

Bloom, H., Hill, C., Black, A., \& Lipsey, M. (2008). Performance trajectories and performance gaps as achievement effect-size. Benchmarks for educational interventions. Journal of Research on Educational Effectiveness, 1(4), 289-328. https://doi.org/10.1080/19345740802400072

Cabezas, V., Paredes, R., Bogolasky, F., Rivero, R., \& Zarhi, M. (2017). First job and the unequal distribution of primary school teachers: Evidence for the case of Chile. Teaching and Teacher Education, 64, 66-78. https://doi.org/10.1016/j.tate.2017.01.017

Castro, J. \& Esposito, B. (2017). The effect of teacher bonuses on learning outcomes and the distribution of teacher skill: Evidence from rural schools in Peru (Working Paper No 104). Lima: Asociación Peruana de Economía.

Centro de Investigación Avanzada en Educación, CIAE. (2017). Evolución de la matrícula en carreras de formación inicial docente (2005-2017). Santiago de Chile: Observatorio Formación Docente, CIAE.

Clotfelter, C., Ladd, H., Vigdor, J., \& Wheeler, J. (2007). High-poverty schools and the distribution of teachers and principals. North Carolina: National Center for Analysis of Longitudinal Data in Education Research.

De Hek, P., De Jong, N., y De Koning, J. (2017). Estudio de proyecciones de oferta y demanda de profesores para los próximos 10 años en Chile. Rotterdam: Erasmus Research \& Business Support (ERBS) y Erasmus University Rotterdam.

De Angelis, K., Presley, J., \& White, B. (2005). The distribution of teacher quality in Illinois. Illinois: Policy Research Report-IERC.

Elige Educar (2014). Factores que inciden en la decisión de estudiar pedagogía (análisis de preferencias). Santiago de Chile: Área de Estudios, Elige Educar.

Elige Educar (2017). Minuta análisis variación matrículas primer año en pedagogía 2016-2017. Santiago de Chile: Área de Estudios, Elige Educar.

Ee-gyeong, K. (2011). Out-of-field secondary school teachers in Korea: Their realities and implications. KEDI Journal of Educational Policy, 8(1), 29-48.

Fuenzalida, D., Ávalos, B., Valenzuela, J. P., y Acuña, F. (agosto, 2014). Trayectorias docentes: ejercicio profesional y abandono. Trabajo presentado en Congreso Interdisciplinario de Investigación en Educación, Santiago de Chile. 
Galvis, L. y Bonilla, L. (2011). Desigualdades regionales en el nivel educativo de los profesores en Colombia. Revista de Economía Institucional, 14(26), 223-240.

Gutiérrez, G., Medeiros, M. P., y Sánchez, M. J. (enero, 2013). Propuesta de mejora a la implementación de la Beca Vocación de Profesor. Trabajo presentado en IV Encuentro Anual de la Sociedad Chilena de Políticas Públicas, Valparaíso, Chile.

Hanushek E. \& Rivkin, S. (2010). Generalizations about using value-added measures of teacher quality. American Economic Review, 100(2), 267271. https://doi.org/10.1257/aer.100.2.267

Hernández, R., Fernández, C., y Baptista. P. (2010). Metodología de la investigación ( $5^{\circ}$ ed.). México: McGraw-Hill.

Kane, T., Rockoff, J., \& Staiger, D. (2006). What does certification tell us about teacher effectiveness? Evidence from New York City. Economics of Education Review, 27(6), 615-631.

Knoeppel, R. (2007). Resource adequacy, equity, and the right to learn: Access to high-quality teachers in Kentucky. Journal of Education Finance, 32(4), 422-442.

Ingersoll, R. (1998). The problem of out-of-field teaching. Phi Delta Kappan, 79(10), 773-776.

Ingersoll, R. (2001). Rejoinder: Misunderstanding the problem of out-offield teaching. Educational Researcher, 30(1), 21-22. https://doi. org/10.3102/0013189x030001021

Ingersoll, R. \& Gruber, K. (1996). Out-of-field teaching and educational equality. Statistical analysis report. Washington D.C.: American Institutes for Research in the Behavioral Sciences.

Jackson, C. (2009). Student demographics, teacher sorting and teacher quality: Evidence from the end of school desegregation. Journal of Labor Economics, 27(2), 213-256. https://doi.org/10.1086/599334

Jerald, C. \& Ingersoll, R. (2002). All talk, no action: Putting an end to out-of-field teaching. Washington D.C.: The Education Trust.

Lu, X., Shen, J., \& Poppink, S. (2007). Are teachers highly qualified? A national study of secondary public-school teachers using SASS 1999-2000. Leadership and Policy in Schools, 6(2), 129-152. https:// doi.org/10.1080/15700760601168636

McConney, A. \& Price, A. (2009). Teaching out-of-field in Western Australia. Australian Journal of Teacher Education, 34(6), 86-100. https://doi. org/10.14221/ajte.2009v34n6.6 
Medeiros, M. P., Gómez, C., Orrego, V., y Sánchez M. S. (2017). Informe Técnico: Idoneidad Docente y Proyección Horas Docentes 2017-2031. Santiago de Chile: Elige Educar.

Ministerio de Educación de Chile, Mineduc. (2008). Marco para la buena enseñanza. Santiago de Chile: Gobierno de Chile.

Ministerio de Educación de Chile, Mineduc. (2014). Evidencias: Aportes a la reflexión sobre movilidad y abandono docente 2013-2014. Santiago de Chile: Gobierno de Chile.

Ministerio de Educación de Chile, Mineduc. (2016). Política Nacional Docente. Santiago de Chile: Gobierno de Chile.

Montoya, A. (2005). Estudio de oferta y demanda de docentes en Chile. Proyección 2004-2015. Santiago de Chile: Mineduc.

Montoya, A. y Blackburn, S. (2010). Estudio de oferta y demanda de docentes en Chile. Proyección 2010-2018. Santiago de Chile: CPEIP y Mineduc.

Nixon, R., Luft, J., \& Ross, R. (2017). Prevalence and predictors of out-of-field teaching in the first five years. Journal of Research in Science Teaching, 54(9), 1197-1218. https://doi.org/10.1002/tea.21402

Organisation for Economic Co-operation and Development, OECD. (2005). Teachers matter: Attracting, developing and retaining effective teachers. Paris: OCDE.

Polizzi, S., Jaggernauth, J., Ray, H., Callahan, B., \& Rushton, G. (2015). Highly qualified or highly unqualified? A longitudinal study of America's public high school biology teachers'. BioScience, 65(8), 812821. https://doi.org/10.1093/biosci/biv093

Rivero, M. (2015). The link of teacher career paths on the distribution of high qualified teachers: A Chilean case study. Education Policy Analysis archives, 23, 73. https://doi.org/10.14507/epaa.v23.1710

Sánchez, M. J., Gutiérrez, G., Hochschild, H., Medeiros, M. P., Ortiz, M., y Sepúlveda, M. J. (2013). Mercado de profesores en el sistema escolar urbano chileno. Calidad en la Educación, 39, 156-194. https://doi.org/10.4067/s0718-45652013000200006

Sanders, W. \& Rivers, J. (1996). Cumulative and residual effects of teachers on future student academic achievement. Tennessee: Value-Added Research and Assessment Center.

Santiago, P. (2002). Teacher demand and supply: Improving teaching quality and addressing teacher shortages. (Education Working Papers, 1). Paris: OECD. http://dx.doi.org/10.1787/232506301033 
Sepúlveda, M. J. (2015). Modificación de la proporción de horas lectivas y no lectivas costos monetarios y repercusión en el mercado docente (Tesis para optar al grado de Magíster en Políticas Públicas), Universidad de Chile, Santiago de Chile.

Schultz, L. (2014). Inequitable dispersion: Mapping the distribution of highly qualified teachers in St. Louis metropolitan elementary schools. Education Policy Analysis Archives, 22(90), 1-20. https://doi.org/10.14507/epaa.v22n90.2014

Valenzuela. J. P. y Sevilla, A. (2013). La movilidad de los nuevos profesores chilenos en la década del 2000: un sistema escolar viviendo en peligro. (Fondecyt $\mathrm{N}^{\circ}$ 1120740). Santiago de Chile: Conicyt.

Zhou, Y. (2012). Out-of-field teaching: A cross-national study on teacher labor market and teacher quality (Tesis para optar al grado de Doctor en Filosofía). Michigan State University, Michigan. 